\title{
Isogeometric shape optimization of nonlinear, curved 3D beams and beam structures
}

\author{
Oliver Weeger ${ }^{\mathrm{a}, \mathrm{b}, *}$, Bharath Narayanan ${ }^{\mathrm{a}}$, Martin L. Dunn ${ }^{\mathrm{a}, \mathrm{c}}$ \\ ${ }^{a}$ Singapore University of Technology and Design, SUTD Digital Manufacturing and Design (DManD) Centre, \\ 8 Somapah Road, Singapore 487372, Singapore \\ ${ }^{b}$ Singapore University of Technology and Design, Information Systems Technology and Design (ISTD) Pillar, \\ 8 Somapah Road, Singapore 487372, Singapore \\ ${ }^{c}$ University of Colorado Denver, College of Engineering and Applied Science, \\ Campus Box 104, Denver, CO 80217-3364, United States
}

\begin{abstract}
Straight beams, rods and trusses are common elements in structural and mechanical engineering, but recent advances in additive manufacturing now also enable efficient freeform fabrication of curved, deformable beams and beam structures, such as microstructures, metamaterials and conformal lattices. To exploit this new design freedom for applications with nonlinear mechanical behavior, we introduce an isogeometric method for shape optimization of curved 3D beams and beam structures. The geometrically exact Cosserat rod theory is used to model nonlinear 3D beams subject to large deformations and rotations. The initial and current geometry are parameterized in terms of NURBS curves describing the beam centerline and an isogeometric collocation approach is used to discretize the strong form of the balance equations. Then, a nonlinear optimization problem is formulated in order to optimize the positions of the control points of the NURBS curve that describes the beam centerline, i.e., the geometry or shape of the beam. To solve the design problem using gradient-based algorithms, we introduce semi-analytical, inconsistent analytical and fully analytical approaches for calculation of design sensitivities. The methods are numerically validated and their performance is investigated, before the applicability and versatility of our 3D beam shape optimization method is illustrated in various numerical applications, including optimization of an auxetic 3D metamaterial.
\end{abstract}

Keywords: Isogeometric analysis, Collocation method, Shape optimization, Nonlinear optimization, 3D beams, Geometrically exact beam theory

\section{Introduction}

Structural design optimization is a well-established and important discipline in computational engineering that aims at optimizing the topology, geometry, shape, sizing, material, microstructure, or support conditions of a structure for a specific objective, such as minimizing its weight or stress concentrations, maximizing its stiffness or dissipation, or matching a certain eigenfrequency or deformation, to name a few [1. General structural optimization techniques such as topological and shape optimization often result in slender structures such as beams, rods and trusses, which are important elements in structural and mechanical engineering anyway. Yet, efficient manufacturing of beam and truss structures is so far largely restricted to assembly of straight and stiff structural elements. However, with the advancement of digital fabrication and additive manufacturing technologies, new possibilities for both design and manufacturing of compliant, slender, free-form structures are emerging [2. Existing and potential applications include active,

\footnotetext{
* Corresponding author

Email addresses: oliver_weeger@sutd.edu.sg (Oliver Weeger), martin.dunn@ucdenver.edu (Martin L. Dunn)
} 


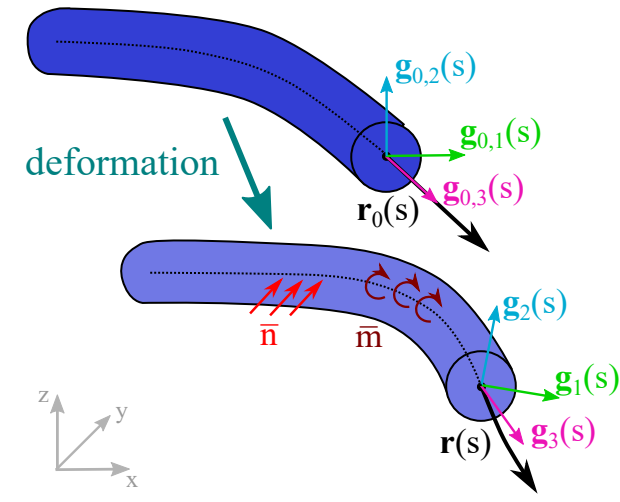

Figure 1: In the geometrically exact $3 \mathrm{D}$ beam model, a beam is defined by its initial shape in terms of the centerline curve $\mathbf{r}_{0}(s)$ and crosssection frame $\mathbf{R}_{0}(s)=\left(\mathbf{g}_{0,1}, \mathbf{g}_{0,2}, \mathbf{g}_{0,3}\right)$. When subject to loading, it deforms into the configuration given by $\mathbf{r}(s)$ and $\mathbf{R}(s)=\left(\mathbf{g}_{1}, \mathbf{g}_{2}, \mathbf{g}_{3}\right)$

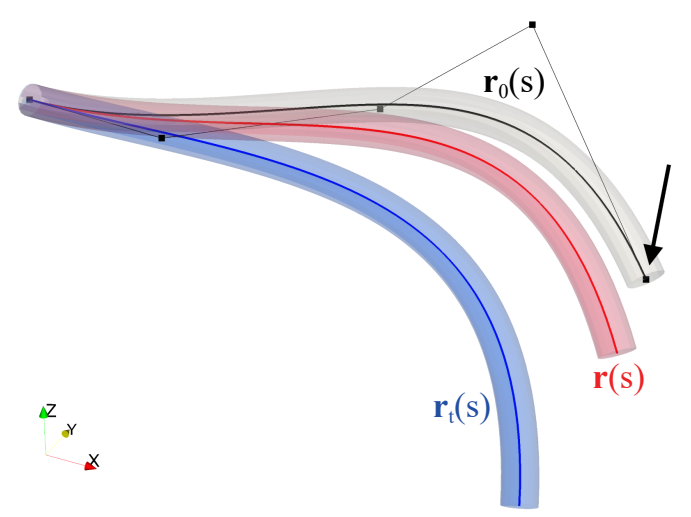

Figure 2: The initial shape of a $3 \mathrm{D}$ beam is defined by the centerline curve $\mathbf{r}_{0}(s)$ (black) and its control points (black squares). The goal of our shape optimization approach is to adjust the initial centerline curve and its control points to minimize a certain mechanical performance criterion, e.g., for the deformed configuration $\mathbf{r}(s)$ (red) to match a given target shape $\mathbf{r}_{t}(s)$ (blue)

self-assembling structures [3-6], smart structures and soft robots [7, 8], deployable composite space structures [9, multi-functional composites [10, 11, advanced metamaterials and microstructures [12 14, cellular structures [15] and conformal lattice structures with slender and curved beam members [16], which all feature compliant, beam-like components that need to be designed with tailored large deformation behavior. This new design freedom thus call for the development of advanced simulation methods and computer-aided engineering tools that can efficiently model beam structures made from soft materials and subject to large deformations, and optimize their design for engineering applications.

Here, we focus on the optimization of beams and beam structures undergoing large elastic deformations, which we model using the geometrically exact 3D beam theory, commonly referred to either as Cosserat rod 17, 18, Reissner 19] or Simo [20 beam theory. It is based on the kinematic description of a beam using its centerline position and the orientation of the rigid cross-section, see Fig. 1. Thus, we aim at optimizing the shape of a beam in terms of its centerline curve. However, the definition of the term shape optimization is somewhat ambiguous, since for $2 \mathrm{D}$ or $3 \mathrm{D}$ continua it typically means optimizing the boundary of a structure, while for 2D structural elements such as shells it means optimizing the geometry as a whole, and for $1 \mathrm{D}$ continua such as beams or rods it its often referred to as optimizing the cross-sectional shape or boundary, whereas for truss structures node positions would be optimized [1, 21, 22. Here, we resort to the interpretation that shape optimization means optimizing the geometry that inherently parameterizes the structure, i.e., in the case of beams the centerline curve, see Fig. 2. While most works in the area of beam or truss structural optimization focus on sizing or cross-sectional design optimization, e.g., for nonlinear space trusses 23] or nonlinear beam buckling [24, few works address this type of shape optimization, e.g., for curved 2D beams 25, 27] and vibrating arches [28, 29, and especially for nonlinear 3D beams it appears to be a largely unexplored field so far. This might be related to two main factors, the lack of applications due to above-mentioned manufacturing limitations for compliant, curved beam structures and the difficulty of deriving and implementing numerical, finite element-based shape optimization formulations for a 3D beam theory that requires higher-order continuity or where not only the centerline curve, but also the cross-section orientations are kinematic variables.

To address this issue, we resort to the concept of isogeometric analysis (IGA) [30, which is based on a consistent description of geometry and numerical solution within computer-aided design (CAD) and computational discretization, e.g., using finite element methods (FEM), typically in terms of B-Spline or NURBS geometries 31. Within the last decade, IGA has impacted virtually all areas of computational mechanics and more recently isogeometric collocation (IGA-C) methods have been introduced [32, 33] as an efficient alternative to finite element methods [34]. So far, IGA-C has been successfully applied especially 
in structural mechanics, to beam [35]38 and shell models 39 41], as well as other areas of continuum mechanics, such as elastostatics and dynamics [42, and large deformation elasticity with contact [43].

IGA has been applied for various design and design optimization problems, such as interactive geometry modeling [44], design space exploration [45], and parametric design optimization [46], but the consistent geometry description using spline functions is particularly beneficial for shape optimization problems, where the control points of the spline geometry directly serve as optimization variables. Thus, remeshing is avoided by directly using the (refined) geometry and function spaces, that are constantly updated throughout an iterative optimization process, within the IGA. This general concept has already been explored in early studies on using B-Splines and NURBS for shape optimization [47 50 and then been exploited in isogeometric structural shape optimization methods [51, 52. An important aspect has been the derivation of sensitivities for gradient-based optimization, whether analytical or semi-analytical 53556 . Applications of isogeometric shape optimization also include linear [26, 29, 57, and nonlinear 27, 2D beams, and more recently also boundary elements and T-Splines [58, triangulations [59, subdivision shell structures [60], as well as auxetic structures via computational homogenization 61. Concurrent shape and material optimization of anisotropic shell structures was presented in [62, along with a discussion of typical issues faced in shape optimization, such as convergence and robustness regarding large shape changes.

In this work, we exploit the advantages of isogeometric discretizations, here an isogeometric collocation method, in terms of a unified geometric parameterization using NURBS and introduce, for the first time, a shape optimization approach for geometrically exact, curved 3D beams and beam structures. A major challenge addressed is the derivation of the gradient, or more specifically the sensitivities required for gradient-based nonlinear optimization using the adjoint method. For geometrically exact 3D beams, this is particularly challenging since the initial orientations of the cross-sections depend implicitly on the centerline curve and the design variables. Here, we derive and investigate a semi-analytical, finite difference approach, see [55, an incomplete analytical method that neglects this dependency, as well as a fully analytical approach that solves another adjoint problem to obtain the sensitivities of initial orientations.

The further structure of this manuscript is as follows: We begin with a brief outline of the geometrically exact 3D beam model, which builds the mechanical foundation of this work, in Sect. 2. Then, we review the isogeometric spline parameterization and collocation method from [38, which is used to numerically discretize the beam model, in Sect. 3. In Section 4, the shape optimization problem is introduced as a constrained, nonlinear optimization problem, where the control points of the centerline curve serve as design variables. The computation of the gradient for gradient-based optimization using the adjoint method and the derivation and evaluation of required sensitivities are outlined in detail. Furthermore, various types of objective functions and constraints for optimization of beams and beam structures are introduced. The semi-analytical, inconsistent analytical and fully analytical sensitivity calculation methods are first validated in Sect. 5along with numerical optimization algorithms. Then, the isogeometric shape optimization method is applied to further numerical examples for optimization of curved beams and an auxetic, 3D lattice-like metamaterial. Finally, we conclude with a summary and an outlook in Sect. 6 .

\section{Geometrically exact 3D beam model}

First, we briefly introduce the mechanical description of slender, elastic, 3-dimensional beams using the geometrically exact Cosserat rod model [17, 18, 20]. The Cosserat rod theory can be seen as a nonlinear extension of the spatial Timoshenko beam model to large deformations and rotations, and is thus also based on the assumption that the cross-sections remain undeformed, but not necessarily normal to the tangent of the centerline curve, accounting for shear deformation.

A rod is represented as a framed curve, see Fig. 1, and its configuration is completely described by its centerline curve $\mathbf{r}$ and an orthonormal basis field, i.e., a frame $\mathbf{R}$ :

$$
\begin{gathered}
\mathbf{r}:[0, L] \rightarrow \mathbb{R}^{3}, \\
\mathbf{R}:[0, L] \rightarrow S O(3) .
\end{gathered}
$$

The centerline curve is arc-length parameterized in the initial configuration given by $\mathbf{r}_{0}$ and $\mathbf{R}_{0}$, i.e., $\left\|\mathbf{r}_{0}^{\prime}(s)\right\|=$ 
$\left\|\frac{d \mathbf{r}_{0}}{d s}\right\|=1 \forall s \in[0, L]$. Thus, $L=\int_{0}^{L}\left\|\mathbf{r}_{0}^{\prime}(s)\right\| d s$ is the length of the curve. The frames represent the evolution of the orientation of the cross-section and can be associated with $3 \mathrm{D}$ rotation matrices $\mathbf{R}(s)=$ $\left(\mathbf{g}_{1}(s), \mathbf{g}_{2}(s), \mathbf{g}_{3}(s)\right) \in \mathbb{R}^{3 \times 3}: \mathbf{R}^{\top} \mathbf{R}=\mathbf{I}, \operatorname{det} \mathbf{R}=1 \forall s \in[0, L]$. As in 38 , we use unit quaternions $\mathbf{q}=\left(q_{1}, q_{2}, q_{3}, q_{4}\right)^{\top} \in \mathbb{R}^{4}:\|\mathbf{q}\|=1$, to parameterize these rotation matrices:

$$
\mathbf{q}:[0, L] \rightarrow S O(3) \leadsto \mathbf{R}(s)=\mathbf{R}(\mathbf{q}(s))
$$

where

$$
\mathbf{R}(\mathbf{q})=\left(\begin{array}{ccc}
q_{1}^{2}-q_{2}^{2}-q_{3}^{2}+q_{4}^{2} & 2\left(q_{1} q_{2}-q_{3} q_{4}\right) & 2\left(q_{1} q_{3}+q_{2} q_{4}\right) \\
2\left(q_{1} q_{2}+q_{3} q_{4}\right) & -q_{1}^{2}+q_{2}^{2}-q_{3}^{2}+q_{4}^{2} & 2\left(q_{2} q_{3}-q_{1} q_{4}\right) \\
2\left(q_{1} q_{3}-q_{2} q_{4}\right) & 2\left(q_{2} q_{3}+q_{1} q_{4}\right) & -q_{1}^{2}-q_{2}^{2}+q_{3}^{2}+q_{4}^{2}
\end{array}\right)
$$

Using the initial, undeformed configuration $\left(\mathbf{r}_{0}(s), \mathbf{R}_{0}(s)\right)$ the kinematics of the current, deformed configuration $(\mathbf{r}(s), \mathbf{R}(s))$ can be derived. Dropping the dependency on the arc-length parameter $s$ in the notation, the translational and rotational strains are defined as:

$$
\begin{aligned}
\varepsilon & =\mathbf{R}^{\top} \mathbf{r}^{\prime}-\mathbf{R}_{0}^{\top} \mathbf{r}_{0}^{\prime}, \\
\kappa & =\mathbf{k}-\mathbf{k}_{0},
\end{aligned}
$$

where $\mathbf{k}$ is the curvature vector of the rod:

$$
\mathbf{k}=\left(\begin{array}{l}
\mathbf{g}_{3}^{\top} \mathbf{g}_{2}^{\prime} \\
\mathbf{g}_{1}^{\top} \mathbf{g}_{3}^{\prime} \\
\mathbf{g}_{2}^{\top} \mathbf{g}_{1}^{\prime}
\end{array}\right) \quad \Leftrightarrow \quad[\mathbf{k}]_{\times}=\mathbf{R}^{\prime \top} \mathbf{R}
$$

and $[\cdot]_{\times}$represents the skew-symmetric cross-product matrix.

Remark 1. Here, we define that the cross-section lies in the plane spanned by $\boldsymbol{g}_{1}$ and $\boldsymbol{g}_{2}$, and thus $\boldsymbol{g}_{3}$ is orthonormal to the cross-section. In the initial configuration, it then holds that $\boldsymbol{R}_{0}^{\top} \boldsymbol{r}_{0}^{\prime}=\boldsymbol{e}_{3}$ if $\boldsymbol{r}_{0}^{\prime}=\boldsymbol{g}_{0,3}$, i.e, if the tangent of the centerline curve is orthogonal to the cross-section. Typically, $\boldsymbol{R}_{0}$ is chosen such that $\boldsymbol{r}_{0}^{\prime}=\boldsymbol{g}_{0,3}$ and then the choice of $\boldsymbol{g}_{0,2}$ and $\boldsymbol{g}_{0,3}$ is arbitrary for a circular cross-section. However, several methods exist to determine the initial cross-section orientations depending on $\boldsymbol{r}_{0}$ and its derivatives, such as the Darboux frame:

$$
\boldsymbol{g}_{0,3}=\boldsymbol{r}_{0}^{\prime}, \quad \boldsymbol{g}_{0,1}=\frac{\boldsymbol{r}_{0}^{\prime \prime}}{\left\|\boldsymbol{r}_{0}^{\prime \prime}\right\|}, \quad \boldsymbol{g}_{0,2}=\boldsymbol{g}_{0,3} \times \boldsymbol{g}_{0,1},
$$

or the rotation-free Bishop frame, which is defined implicitly through:

$$
\boldsymbol{g}_{0,3}=\boldsymbol{r}_{0}^{\prime}, \quad \boldsymbol{k}_{0,3}=0 \quad \Leftrightarrow \quad \boldsymbol{g}_{0,1}^{\top} \boldsymbol{r}_{0}^{\prime}=0, \quad \boldsymbol{g}_{0,2}^{\top} \boldsymbol{r}_{0}^{\prime}=0, \quad \boldsymbol{g}_{0,2}^{\top} \boldsymbol{g}_{0,1}^{\prime}=0 .
$$

While the former is not defined for straight rods with $\boldsymbol{r}_{0}^{\prime \prime}=\boldsymbol{O}$, the latter is only defined implicitly through the conditions given. Thus, sophisticated heuristics have to be applied to ensure that the definition of the frame remains $C^{1}$ when the curvature $\boldsymbol{r}_{0}^{\prime \prime}$ vanishes or changes rapidly.

With a commonly used linear elastic constitutive law, which restricts the model to small strains and stresses, but nevertheless allows large deformations and rotations, the translational and rotational stress resultants are computed as:

$$
\begin{aligned}
& \boldsymbol{\sigma}=\mathbf{A} \boldsymbol{\varepsilon}+\mathbf{B} \boldsymbol{\kappa}, \\
& \boldsymbol{\chi}=\mathbf{B}^{\top} \varepsilon+\mathbf{C} \boldsymbol{\kappa} .
\end{aligned}
$$

Here, we focus on homogeneous, circular cross-sections with radius $r$, for which constitutive matrices $\mathbf{A}, \mathbf{B}, \mathbf{C} \in \mathbb{R}^{3 \times 3}$ take the following form:

$$
\mathbf{A}=\left(\begin{array}{ccc}
k_{1} G A & 0 & 0 \\
0 & k_{2} G A & 0 \\
0 & 0 & E A
\end{array}\right), \quad \mathbf{B} \equiv \mathbf{0}, \quad \mathbf{C}=\left(\begin{array}{ccc}
E I_{1} & 0 & 0 \\
0 & E I_{2} & 0 \\
0 & 0 & G J_{P}
\end{array}\right)
$$


where $A=\pi r^{2}$ is the area of the cross-section, $I_{1}=I_{2}=\frac{1}{12} \pi r^{4}$ are the second moments of area, $J_{P}=\frac{1}{6} \pi r^{4}$ is the polar moment of area, and $k_{1}=k_{2}=\frac{5}{6}$ is the shear correction factor. Furthermore, $E$ is the Young's modulus, $G=E /(1+2 \nu)$ the shear modulus and $\nu$ the Poisson's ratio of the material.

The stress vectors defined in (8) are then transformed from the material to the spatial configuration to obtain the vectors of internal forces $\mathbf{n}$ and moments $\mathbf{m}$ :

$$
\begin{array}{r}
\mathbf{n}=\mathbf{R} \boldsymbol{\sigma}, \\
\mathbf{m}=\mathbf{R} \chi
\end{array}
$$

Then, the equilibrium equations of linear and angular momentum are formulated in the current, spatial configuration:

$$
\begin{aligned}
\mathbf{n}^{\prime}+\overline{\mathbf{n}}=\mathbf{0} & \forall s \in(0, L), \\
\mathbf{m}^{\prime}+\mathbf{r}^{\prime} \times \mathbf{n}+\overline{\mathbf{m}}=\mathbf{0} & \forall s \in(0, L),
\end{aligned}
$$

where $\overline{\mathbf{n}}$ and $\overline{\mathbf{m}}$ are externally applied distributed forces and moments. These differential equations have to be completed with boundary conditions at the ends of the rod for $s=0$ and $s=L$. Prescribed displacements $\hat{\mathbf{r}}$ and rotations $\hat{\mathbf{q}}$ are specified as Dirichlet boundary conditions $\mathbf{r}(s)=\hat{\mathbf{r}}, \mathbf{q}(s)=\hat{\mathbf{q}}$ at $s=0, L$ and forces $\hat{\mathbf{n}}$ and moments $\hat{\mathbf{m}}$ as Neumann boundary conditions $\mathbf{n}(s)=\hat{\mathbf{n}}, \mathbf{m}(s)=\hat{\mathbf{m}}$ at $s=0, L$. Additionally, a normalization constraint for the quaternions must hold to complete the equilibrium equations:

$$
\mathbf{q}^{\top} \mathbf{q}-1=0 \quad \forall s \in[0, L]
$$

\section{Isogeometric parameterization and collocation}

For the discretization of the geometrically exact beam model and its numerical solution we resort to the concept of isogeometric analysis and isogeometric collocation methods. Isogeometric analysis is based on the parameterization of the initial geometry and the unknown solution fields using spline functions. Then, we use a collocation method to determine the coefficients of the solution. This method was already introduced for the Cosserat rod model in 38 , and here we briefly outline the approach.

\subsection{Spline parameterization of $3 D$ beams}

The basis of isogeometric methods is the parameterization of geometry and unknowns using spline functions, such as B-Splines and Non-Uniform Rational B-Splines (NURBS), which are commonly employed to describe geometries in computer-aided design [30. Here, we briefly review the terminology and properties of B-Splines and NURBS, while detailed definitions and algorithms can be found in [31.

B-Splines are piece-wise polynomial functions and NURBS piece-wise rational function of degree $p$ and order $p+1$. The spline (B-Spline or NURBS) basis functions $N_{i}(\xi): \Omega_{0} \rightarrow[0,1], i=1, \ldots, n$ are defined on a parameter domain $\Omega_{0}=\left[\xi_{1}, \xi_{m}\right] \subset \mathbb{R}$, typically $\Omega_{0}=[0,1]$, using a knot vector $\Xi=\left\{\xi_{1}, \ldots, \xi_{m}\right\}$ with $m=n+p+1$, i.e., a non-decreasing sequence of knots $\xi_{i} \in \mathbb{R}(i=1, \ldots, m), \xi_{i} \leq \xi_{i+1}(i=1, \ldots, m-1)$. For two distinct knots $\xi_{i} \neq \xi_{i+1}$ the half-open interval $\left[\xi_{i}, \xi_{i+1}\right)$ is called the $i$-th knot span or element and the total number of nonzero knot spans or elements in $\Xi$ is denoted by $\ell$. Typically, only open knot vectors that are interpolatory at $\xi_{1}$ and $\xi_{m}$ are used in IGA, which means that the first and last knot have multiplicity $p+1$, while inner knots have multiplicity $1 \leq k \leq p$.

The geometry of a Cosserat rod can then be expressed using spline curves for the initial centerline curve $\mathbf{r}_{0}$ and the quaternion curve $\mathbf{q}_{0}$ parameterizing the initial frame $\mathbf{R}_{0}$ :

$$
\begin{aligned}
& \mathbf{r}_{0}: \Omega_{0} \rightarrow \mathbb{R}^{3}, \quad \mathbf{r}_{0}(\xi)=\sum_{i=1}^{n_{0}} N_{0, i}(\xi) \mathbf{r}_{0, i}, \\
& \mathbf{q}_{0}: \Omega_{0} \rightarrow \mathbb{R}^{4}, \quad \mathbf{q}_{0}(\xi)=\sum_{i=1}^{n_{0}} N_{0, i}(\xi) \mathbf{q}_{0, i}, \quad\left\|\mathbf{q}_{0}(\xi)\right\|=1
\end{aligned}
$$




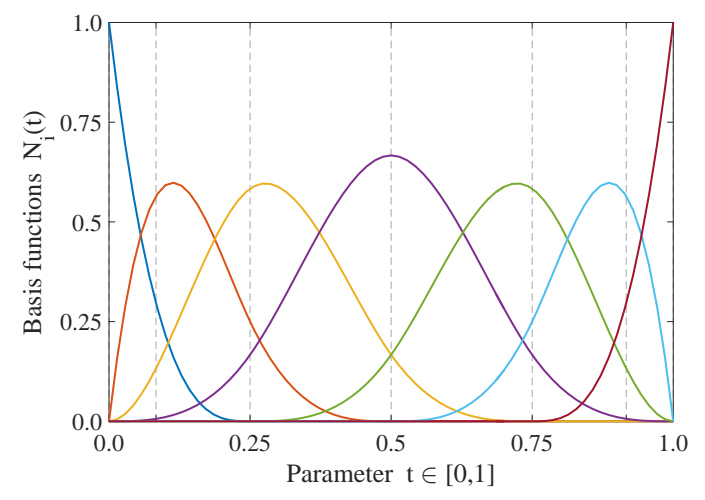

(a) B-Spline basis functions

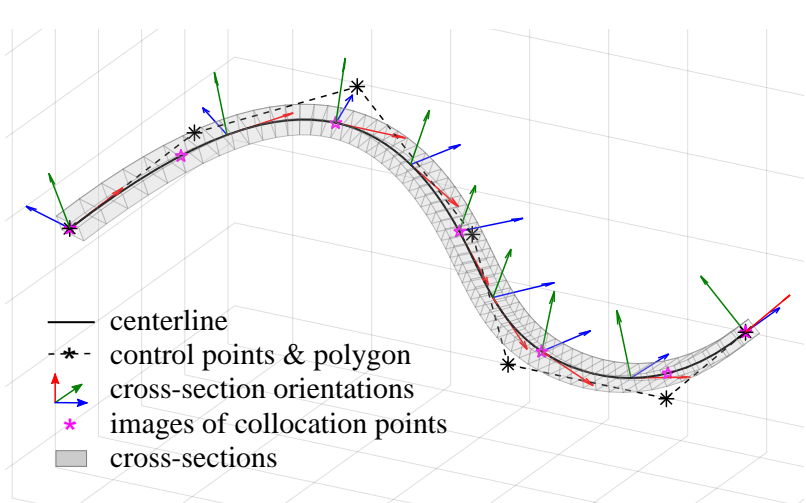

(b) Isogeometric parameterization of rod with B-Spline curve

Figure 3: Isogeometric rod parameterization; cubic B-Spline basis functions as shown in (a) with $p=3, m=11, n=7, \Xi=$ $\left\{0,0,0,0, \frac{1}{4}, \frac{1}{2}, \frac{3}{4}, 1,1,1,1\right\}$ are used for the isogeometric parameterization of the Cosserat rod in (b) with centerline and rotation quaternions as B-Spline curves (see [38])

with control points $\mathbf{r}_{0, i} \in \mathbb{R}^{3}, \mathbf{q}_{0, i} \in \mathbb{R}^{4}\left(i=1, \ldots, n_{0}\right)$. Here, we chose $\mathbf{r}_{0}$ and $\mathbf{q}_{0}$ to be parameterized with same space of spline functions $\left\{N_{0, i}\right\}$, as this choice results in optimal convergence orders, see [38].

For illustration, the parameterization of a rod using cubic B-Spline basis functions $(p=3)$ with $n=7$ control points and $\ell=4$ elements in the knot vector $\Xi=\left\{0,0,0,0, \frac{1}{4}, \frac{1}{2}, \frac{3}{4}, 1,1,1,1\right\}$ is shown in Fig. 3 . Figure $3 \mathrm{a}$ shows the basis functions and Fig. $3 \mathrm{~b}$ the rod itself in terms of its centerline curve and crosssection frames.

Since the initial centerline curve is parameterized as a spline curve $\mathbf{r}_{0}(\xi): \Omega_{0} \rightarrow \mathbb{R}^{3}$ with an arbitrary domain of parameterization $\Omega_{0} \subset \mathbb{R}$, it is general not arc-length parameterized. Thus, the arc-length derivatives $\mathbf{r}_{0}^{\prime}$ and $\mathbf{r}_{0}^{\prime \prime}$ need to be expressed in terms of the parametric spline derivatives $\dot{\mathbf{r}}_{0}=\frac{d \mathbf{r}_{0}}{d \xi}$ and $\ddot{\mathbf{r}}_{0}=\frac{d^{2} \mathbf{r}_{0}}{d \xi^{2}}$

$$
\begin{aligned}
& \mathbf{r}_{0}^{\prime}(\xi)=\frac{d \mathbf{r}_{0}}{d s}=\frac{d \mathbf{r}_{0}}{d \xi} \frac{d \xi}{d s}=\dot{\mathbf{r}}_{0}\left(\frac{d s}{d \xi}\right)^{-1}=\frac{1}{J} \dot{\mathbf{r}}_{0} \\
& \mathbf{r}_{0}^{\prime \prime}(\xi)=\frac{d^{2} \mathbf{r}_{0}}{d s^{2}}=\frac{d}{d s}\left[\frac{1}{J} \dot{\mathbf{r}}_{0}\right] \frac{d}{d \xi}\left[\frac{1}{J} \dot{\mathbf{r}}_{0}\right] \frac{d \xi}{d s}=\frac{1}{J^{2}} \ddot{\mathbf{r}}_{0}-\frac{\dot{\mathbf{r}}_{0}^{T} \ddot{\mathbf{r}}_{0}}{J^{4}} \dot{\mathbf{r}}_{0}
\end{aligned}
$$

with the Jacobi determinant of the parameterization:

$$
J(\xi):=\frac{d s}{d \xi}=\frac{d}{d \xi}\left[\int_{0}^{\xi}\left\|\dot{\mathbf{r}}_{0}(\zeta)\right\| d \zeta\right]=\left\|\dot{\mathbf{r}}_{0}(\xi)\right\| .
$$

Similarly, the derivatives of any vector field $\xi \rightarrow \mathbf{y}(\xi): \Omega_{0} \rightarrow \mathbb{R}^{d}$ depending on the spline parameterization can be converted to arc-length parameterization using:

$$
\mathbf{y}^{\prime}=\frac{1}{J} \dot{\mathbf{y}}, \quad \mathbf{y}^{\prime \prime}=\frac{1}{J^{2}} \ddot{\mathbf{y}}-\frac{\dot{\mathbf{r}}_{0}^{T} \ddot{\mathbf{r}}_{0}}{J^{4}} \dot{\mathbf{y}}
$$




\subsection{Discretization and collocation of equilibrium equations}

The unknown centerline positions $\mathbf{r}$ and quaternions $\mathbf{q}$ in the current, deformed configuration are then also discretized and approximated as spline curves $\mathbf{r}_{h} \approx \mathbf{r}$ and $\mathbf{q}_{h} \approx \mathbf{q}$ :

$$
\begin{aligned}
& \mathbf{r}_{h}: \Omega_{0} \rightarrow \mathbb{R}^{3}, \quad \mathbf{r}_{h}(\xi)=\sum_{i=1}^{n} N_{i}(\xi) \mathbf{r}_{i}, \\
& \mathbf{q}_{h}: \Omega_{0} \rightarrow \mathbb{R}^{4}, \quad \mathbf{q}_{h}(\xi)=\sum_{i=1}^{n} N_{i}(\xi) \mathbf{q}_{i}, \quad\left\|\mathbf{q}_{h}(\xi)\right\|=1,
\end{aligned}
$$

with control points $\mathbf{r}_{i} \in \mathbb{R}^{3}, \mathbf{q}_{i} \in \mathbb{R}^{4}(i=1, \ldots, n)$. The basis functions $\left\{N_{i}\right\}$ refer to either the same or typically a $p$ - $/ h$ - $/ k$-refined version of $\left\{N_{0, i}\right\}$ from 13 .

Then, the strong form of the equilibrium equations of linear and angular momentum is collocated, i.e., the discretization from (17) is substituted into the balance equations (11) and the quaternion normalization condition 12 , which are then evaluated at a set of collocation points $\tau_{i} \in \Omega_{0}, i=2, \ldots, n-1$ :

$$
\begin{array}{rlrl}
\mathbf{f}_{\mathbf{n}}\left(\tau_{i}\right) & :=\mathbf{n}^{\prime}\left(\tau_{i}\right)+\overline{\mathbf{n}}\left(\tau_{i}\right) & =\mathbf{0}, \\
\mathbf{f}_{\mathbf{m}}\left(\tau_{i}\right) & :=\mathbf{m}^{\prime}\left(\tau_{i}\right)+\mathbf{r}_{h}^{\prime}\left(\tau_{i}\right) \times \mathbf{n}\left(\tau_{i}\right)+\overline{\mathbf{m}}\left(\tau_{i}\right) & =\mathbf{0}, \\
\mathbf{f}_{\mathbf{q}}\left(\tau_{i}\right) & :=\mathbf{q}_{h}\left(\tau_{i}\right)^{\top} \mathbf{q}_{h}\left(\tau_{i}\right)-1 & & =0 .
\end{array}
$$

At the boundary, i.e., for $i=1$ and $i=n$, the above-mentioned equations are replaced with the evaluations of the boundary conditions. To guarantee the stability of the method [32], the collocation points are chosen as the Greville abscissae of the spline knot vector $\Xi$, which are defined as:

$$
\tau_{i}=\frac{\xi_{i+1}+\ldots+\xi_{i+p}}{p}, \quad i=1, \ldots, n .
$$

Alternatively, so-called superconvergence points could be used as collocation points [63, 64.

Equation (18) defines a nonlinear system of $n_{x}=7 n$ equations for the $7 n$ unknowns summarized in the state vector $\overrightarrow{\mathbf{x}}=(\overrightarrow{\mathbf{r}}, \overrightarrow{\mathbf{q}})$, with the control point vectors $\overrightarrow{\mathbf{r}}=\left(\mathbf{r}_{i}\right)_{i=1, \ldots, n}$ for $\mathbf{r}_{h}$ and $\overrightarrow{\mathbf{q}}=\left(\mathbf{q}_{i}\right)_{i=1, \ldots, n}$ for $\mathbf{q}_{h}$, which we can be summarized in the nonlinear force residual vector $\overrightarrow{\mathbf{f}}: \mathbb{R}^{7 n} \rightarrow \mathbb{R}^{7 n}$ :

$$
\overrightarrow{\mathbf{f}}(\overrightarrow{\mathbf{x}})=\left(\begin{array}{c}
\mathbf{f}_{\mathrm{n}}\left(\tau_{i}\right) \\
\mathbf{f}_{\mathrm{m}}\left(\tau_{i}\right) \\
\mathbf{f}_{\mathrm{q}}\left(\tau_{i}\right)
\end{array}\right){ }_{i=1, \ldots, n}(\overrightarrow{\mathbf{x}})=\mathbf{0}
$$

This nonlinear system then has to be solved to determine the deformed configuration of the beam, e.g., with Newton's method or arc-length continuation, which require several incremental (load) steps and the evaluation of the tangent stiffness matrix $\mathbf{K}(\overrightarrow{\mathbf{x}})=d \overrightarrow{\mathbf{f}} / d \overrightarrow{\mathbf{x}}$ for linearization, see 38 for details.

In 38, it was furthermore shown how the isogeometric collocation formulation can be extended to rod structures, where several rods can be interconnected at their ends. The rods are coupled rigidly, which means that change of position and rotation have to be equal for all $n_{I}$ rods connected at an interface and that the equilibria of linear and angular momentum must hold at the interface. To enforce these conditions, the forces at all collocation points that meet at the interface are summed up and inserted into the rows corresponding to the collocation point of the first rod at the interface:

$$
\begin{aligned}
& \mathbf{f}_{\mathrm{n}}^{(1)} \leftarrow \sum_{k=1}^{n_{I}} I_{k} \mathbf{f}_{\mathrm{n}}^{(k)}, \\
& \mathbf{f}_{\mathrm{m}}^{(1)} \leftarrow \sum_{k=1}^{n_{I}} I_{k} \mathbf{f}_{\mathrm{m}}^{(k)},
\end{aligned}
$$


where $I_{k}=-1$ if it is the first collocation point $(\xi=0)$ and $I_{k}=1$ if it is the last collocation point $(\xi=1)$ of the rod $k$ that is attached to the interface. The equilibrium equations at the other interfacing collocation points for $k=2, \ldots, n_{I}$ are replaced with equality conditions for centerline positions and change of rotation quaternions:

$$
\begin{aligned}
& \mathbf{f}_{\mathrm{n}}^{(k)} \leftarrow \mathbf{r}^{(1)}-\mathbf{r}^{(k)}, \\
& \mathbf{f}_{\mathrm{m}}^{(k)} \leftarrow \mathbf{q}^{(1)} \overline{\mathbf{q}}_{0}^{(1)}-\mathbf{q}^{(k)} \overline{\mathbf{q}}_{0}^{(k)} .
\end{aligned}
$$

This isogeometric collocation method for beams and beam structures has been implemented into a computer program using the G+Smo (Geometry + Simulation Modules) 65] open-source C++ library for isogeometric analysis, see 38 .

\section{Isogeometric shape optimization}

Now we combine the isogeometric spline parameterizations and discretizations of geometrically exact 3D beams with a shape optimization approach. In this way, we seek to determine the shape of a beam in terms of its initial centerline curve $\mathbf{r}_{0}$ such that it is optimal for a given objective function and constraints that capture the mechanical behavior and performance of the beam.

\subsection{Problem formulation}

In Sect. 3.1 we have already introduced the parameterization of the initial centerline curve $\mathbf{r}_{0}$ as a B-Spline or NURBS curve:

$$
\mathbf{r}_{0}: \Omega_{0} \rightarrow \mathbb{R}^{3}, \quad \mathbf{r}_{0}(\xi)=\sum_{i=1}^{n_{0}} N_{0, i}(\xi) \mathbf{r}_{0, i}
$$

The main variables that describe this curve and thus define the shape of the beam are its control points $\mathbf{r}_{0, i}$, which we summarize into a vector of design variables $\overrightarrow{\mathbf{u}}=\left(\mathbf{r}_{0, i}\right)_{i=1, \ldots, n_{0}} \in \mathbb{R}^{n_{u}}$. Now, we introduce the mathematical formulation of a nonlinear optimization 66 problem with design vector $\overrightarrow{\mathbf{u}}$ :

$$
\begin{array}{ll}
\min _{\overrightarrow{\mathbf{u}}} & g(\overrightarrow{\mathbf{u}}, \overrightarrow{\mathbf{x}}(\overrightarrow{\mathbf{u}})) \\
\text { s.t. } & \overrightarrow{\mathbf{f}}(\overrightarrow{\mathbf{x}} ; \overrightarrow{\mathbf{u}})=\mathbf{0}, \\
& \overrightarrow{\mathbf{u}}_{0} \leq \overrightarrow{\mathbf{u}} \leq \overrightarrow{\mathbf{u}}_{1}, \\
& \overrightarrow{\mathbf{h}}(\overrightarrow{\mathbf{u}}) \leq \mathbf{0} .
\end{array}
$$

Here, $g: \mathbb{R}^{n_{u}} \times \mathbb{R}^{n_{x}} \rightarrow \mathbb{R}$ is a scalar objective function that will be minimized and typically depends either explicitly or at least implicitly on both, the design vector $\overrightarrow{\mathbf{u}}$ and the state vector $\overrightarrow{\mathbf{x}}$. The nonlinear equality constraint $\overrightarrow{\mathbf{f}}: \mathbb{R}^{n_{u}} \times \mathbb{R}^{n_{x}} \rightarrow \mathbb{R}^{n_{x}}$, i.e., the nonlinear force residual vector given in $(20)$, defines the implicit dependency of the state vector $\overrightarrow{\mathbf{x}}(\overrightarrow{\mathbf{u}})$ on the design vector, i.e., the dependency of the structural response of the beam on its initial shape. Furthermore, $\overrightarrow{\mathbf{u}}_{0}, \overrightarrow{\mathbf{u}}_{1} \in \mathbb{R}^{n_{u}}$ define the lower and upper bounds of each design variable and $\overrightarrow{\mathbf{h}}: \mathbb{R}^{n_{u}} \rightarrow \mathbb{R}^{n_{h}}$ defines any additional nonlinear inequality constraints on the design variables.

To solve the nonlinear, constrained optimization problem given by (24), two general types of algorithms can be distinguished, global derivative-free or local gradient-based methods 66 68. While the advantages of the former are that they do not require implementation and evaluation of gradients and that they avoid local minima by searching the whole design space, the severe disadvantages in the context of nonlinear structural shape optimization are that convergence is slower, requiring many expensive function evaluations and, most importantly, that trial design vectors $\overrightarrow{\mathbf{u}}$ may often lead to mechanically infeasible designs. On the other hand, gradient-based methods are faster, typically converging quadratically and thus requiring less function evaluations, but they can only find local minima, depending strongly on the convexity of the objective function, and require the implementation and evaluation of gradients. Here, we generally use the local, gradient-based algorithms provided by the C++ implementation of the NLopt nonlinear optimization library 69 . 
The solution of the shape optimization problem (24) using a gradient-based algorithm interfacing with the isogeometric collocation method requires the following steps within each optimization step:

1. Candidate design vector $\overrightarrow{\mathbf{u}}$ is received from optimizer and used to update $\mathbf{r}_{0}$.

2. Quaternions for initial cross-section orientations $\mathbf{q}_{0}\left(\mathbf{r}_{0}\right)$ are determined, see Remark 1 .

3. State vector $\overrightarrow{\mathbf{x}}$ is obtained by solving $\overrightarrow{\mathbf{f}}(\overrightarrow{\mathbf{x}})=\mathbf{0}$, see 20 .

4. Objective function $g(\overrightarrow{\mathbf{u}}, \overrightarrow{\mathbf{x}})$ and gradient $\frac{D g}{D \overrightarrow{\mathbf{u}}}$ are evaluated, see Sect. 4.2 and 4.3

5. Inequality constraints $\overrightarrow{\mathbf{h}}(\overrightarrow{\mathbf{u}})$ and gradient $\frac{d \mathbf{h}}{d \overrightarrow{\mathbf{u}}}$ are evaluated, see Sect. 4.3 .

6. Function values and gradients are returned to optimizer.

\subsection{Gradient and sensitivities}

As mentioned above, an important step in solving a nonlinear optimization problem using a gradientbased method is the evaluation of the gradient of the objective function with respect to the design variables. However, in any type of structural design optimization problem, this gradient depends on the design variables also implicitly through the state variables:

$$
\frac{D g}{D \overrightarrow{\mathbf{u}}}=\frac{d g}{d \overrightarrow{\mathbf{u}}}+\frac{d g}{d \overrightarrow{\mathbf{x}}} \frac{d \overrightarrow{\mathbf{x}}}{d \overrightarrow{\mathbf{u}}}
$$

The explicit derivatives of $g$ w.r.t. $\overrightarrow{\mathbf{u}}$ and $\overrightarrow{\mathbf{x}}$ can be directly derived from the definition of the objective function and hence easily be computed, see Sect. 4.3 , but the derivatives of $\overrightarrow{\mathbf{x}}$ w.r.t. $\overrightarrow{\mathbf{u}}$ are more difficult to obtain.

The simplest way of evaluating the gradient (25) is thus a numerical, finite difference approach. However, this requires $n_{u}$ additional evaluations of $g$ for a first-order finite difference method and is thus very inefficient, especially in the context of nonlinear mechanics, where the nonlinear system 20 has to be solved for every evaluation. Here, we use the adjoint method [66] to compute the implicit derivative $\frac{d g}{d \overrightarrow{\mathbf{x}}} \frac{d \overrightarrow{\mathbf{x}}}{d \overrightarrow{\mathbf{u}}}$ : Since the constraint $\overrightarrow{\mathbf{f}}(\overrightarrow{\mathbf{x}} ; \overrightarrow{\mathbf{u}})=\mathbf{0}$ has to be satisfied for every design $\overrightarrow{\mathbf{u}}$, it follows that it is stationary, i.e.:

$$
\mathbf{0} \equiv \frac{D \overrightarrow{\mathbf{f}}}{D \overrightarrow{\mathbf{u}}}=\frac{d \overrightarrow{\mathbf{f}}}{d \overrightarrow{\mathbf{u}}}+\frac{d \overrightarrow{\mathbf{f}}}{d \overrightarrow{\mathbf{x}}} \frac{d \overrightarrow{\mathbf{x}}}{d \overrightarrow{\mathbf{u}}} \Rightarrow \frac{d \overrightarrow{\mathbf{f}}}{d \overrightarrow{\mathbf{u}}}=-\frac{d \overrightarrow{\mathbf{f}}}{d \overrightarrow{\mathbf{x}}} \frac{d \overrightarrow{\mathbf{x}}}{d \overrightarrow{\mathbf{u}}}
$$

and thus:

$$
\frac{d g}{d \overrightarrow{\mathbf{x}}} \frac{d \overrightarrow{\mathbf{x}}}{d \overrightarrow{\mathbf{u}}}=-\frac{d g}{d \overrightarrow{\mathbf{x}}}\left(\frac{d \overrightarrow{\mathbf{f}}}{d \overrightarrow{\mathbf{x}}}\right)^{-1} \frac{d \overrightarrow{\mathbf{f}}}{d \overrightarrow{\mathbf{u}}}=-\boldsymbol{\lambda}^{\top} \frac{d \overrightarrow{\mathbf{f}}}{d \overrightarrow{\mathbf{u}}},
$$

where $\boldsymbol{\lambda} \in \mathbb{R}^{n}$ is obtained by solving the linear system :

$$
\frac{d \overrightarrow{\mathbf{f}}^{\top}}{d \overrightarrow{\mathbf{x}}} \quad \boldsymbol{\lambda}={\frac{d g^{\top}}{d \overrightarrow{\mathbf{x}}}}^{\top}
$$

Here, $\frac{d \overrightarrow{\mathbf{f}}}{d \overrightarrow{\mathbf{x}}}=\mathbf{K}(\overrightarrow{\mathbf{x}} ; \overrightarrow{\mathbf{u}})$ is the tangent stiffness matrix and $\frac{d \overrightarrow{\mathbf{f}}}{d \overrightarrow{\mathbf{u}}}=\mathbf{K}_{u}(\overrightarrow{\mathbf{x}} ; \overrightarrow{\mathbf{u}})$ is the sensitivity matrix of the nonlinear force residual vector. $\mathbf{K}_{u}$ can either be determined

a) by a finite difference method, which requires $n_{u}$ additional assemblies of $\overrightarrow{\mathbf{f}}$ for first-order accuracy, thus yielding overall a semi-analytical approach to compute the gradient vector $\frac{D g}{D \overrightarrow{\mathbf{u}}}$, see [55], or

b) by deriving and evaluating $\frac{d \overrightarrow{\mathbf{f}}}{d \overrightarrow{\mathbf{u}}}$ analytically, see the following subsections. 


\subsubsection{Analytical sensitivity calculation}

In the following we provide the detailed derivation of the sensitivities of the nonlinear force vector $\overrightarrow{\mathbf{f}}$ with respect to the design vector $\overrightarrow{\mathbf{u}}=\left(\mathbf{r}_{0, i}\right)_{i=1, \ldots, n_{0}}$, i.e., the sensitivity matrix $\mathbf{K}_{u}=\frac{d \overrightarrow{\mathbf{f}}}{d \overrightarrow{\mathbf{u}}}$. The starting point is, of course, the parameterization of the initial centerline as a spline curve given in $(23)$, and subsequently we derive the partial derivatives of any required quantity w.r.t. a control point $\mathbf{r}_{0, i}$.

For the centerline curve and its parametric derivatives, it trivially follows from (23) that:

$$
\frac{\partial \mathbf{r}_{0}}{\partial \mathbf{r}_{0, i}}=N_{0, i}, \quad \frac{\partial \dot{\mathbf{r}}_{0}}{\partial \mathbf{r}_{0, i}}=\dot{N}_{0, i}, \quad \frac{\partial \ddot{\mathbf{r}}_{0}}{\partial \mathbf{r}_{0, i}}=\ddot{N}_{0, i}
$$

However, we require the arc-length derivatives of the centerline, $\mathbf{r}_{0}^{\prime}$ and $\mathbf{r}_{0}^{\prime \prime}$, see (14), and also the arc-length derivatives of any other vector-valued quantity, $\mathbf{y}^{\prime}$ and $\mathbf{y}^{\prime \prime}$, see $(16)$, and therefore the Jacobian $J$ (15):

$$
\begin{aligned}
\frac{\partial J}{\partial \mathbf{r}_{0, i}} & =\frac{1}{J} \dot{N}_{0, i} \dot{\mathbf{r}}_{0}^{\top}=J N_{0, i}^{\prime} \mathbf{r}_{0}^{\top \top}, \\
\frac{\partial \mathbf{r}_{0}^{\prime}}{\partial \mathbf{r}_{0, i}} & =\frac{1}{J} \dot{N}_{0, i}+\dot{\mathbf{r}}_{0}\left(\frac{-1}{J^{2}} \frac{\partial J}{\partial \mathbf{r}_{0, i}}\right)=N_{0, i}^{\prime}\left(\mathbf{I}-\mathbf{r}_{0}^{\prime} \mathbf{r}_{0}^{\prime \top}\right), \\
\frac{\partial \mathbf{r}_{0}^{\prime \prime}}{\partial \mathbf{r}_{0, i}} & =\frac{1}{J^{2}} \ddot{N}_{0, i}+\ddot{\mathbf{r}}_{0}\left(\frac{-2}{J^{3}} \frac{d \partial J}{\partial \mathbf{r}_{0, i}}\right)-\frac{\dot{\mathbf{r}}_{0}^{\top} \ddot{\mathbf{r}}_{0}}{J^{4}} \dot{N}_{0, i}-\frac{\dot{\mathbf{r}}_{0}}{J^{4}}\left(\dot{\mathbf{r}}_{0}^{\top} \ddot{N}_{0, i}+\dot{\mathbf{r}}_{0}^{\top} \dot{N}_{0, i}\right)-\left(\dot{\mathbf{r}}_{0}^{\top} \ddot{\mathbf{r}}_{0}\right) \dot{\mathbf{r}}_{0}\left(\frac{-4}{J^{5}} \frac{\partial J}{\partial \mathbf{r}_{0, i}}\right) \\
& =N_{0, i}^{\prime \prime} \mathbf{I}-2 N_{0, i}^{\prime} \mathbf{r}_{0}^{\prime \prime} \mathbf{r}_{0}^{\prime}{ }^{\top}-\mathbf{r}_{0}^{\prime}\left(N_{0, i}^{\prime} \mathbf{r}_{0}^{\prime \prime \top}+N_{0, i}^{\prime \prime} \mathbf{r}_{0}^{\prime \top}\right), \\
\frac{\partial \mathbf{y}^{\prime}}{\partial \mathbf{r}_{0, i}} & =\dot{\mathbf{y}}\left(\frac{-1}{J^{2}} \frac{\partial J}{\partial \mathbf{r}_{0, i}}\right)=-N_{0, i}^{\prime} \mathbf{y}^{\prime} \mathbf{r}_{0}^{\prime \top}, \\
\frac{\partial \mathbf{y}_{0, i}^{\prime \prime}}{} & =\ddot{\mathbf{y}}\left(\frac{-2}{J^{3}} \frac{\partial J}{\partial \mathbf{r}_{0, i}}\right)-\left(\dot{\mathbf{r}}_{0}^{\top} \ddot{\mathbf{r}}_{0}\right) \dot{\mathbf{y}}\left(\frac{-4}{J^{5}} \frac{\partial J}{\partial \mathbf{r}_{0, i}}\right)-\frac{\dot{\mathbf{y}}}{J^{4}}\left(\dot{\mathbf{r}}_{0}^{\top} \ddot{N}_{0, i}+\dot{\mathbf{r}}_{0}^{\top} \dot{N}_{0, i}\right) \\
& =-2 N_{0, i}^{\prime} \mathbf{y}^{\prime \prime} \mathbf{r}_{0}^{\prime}-\mathbf{y}^{\prime}\left(N_{0, i}^{\prime} \mathbf{r}_{0}^{\prime \prime \top}+N_{0, i}^{\prime \prime} \mathbf{r}_{0}^{\prime \top}\right) .
\end{aligned}
$$

Now, the sensitivities of the translational and rotational strains, see (4) and their arc-length derivatives can be calculated:

$$
\begin{aligned}
& \frac{\partial \varepsilon}{\partial \mathbf{r}_{0, i}}=\mathbf{R}^{\top} \frac{\partial \mathbf{r}^{\prime}}{\partial \mathbf{r}_{0, i}}-\mathbf{R}_{0}^{\top} \frac{\partial \mathbf{r}_{0}^{\prime}}{\partial \mathbf{r}_{0, i}}-\frac{\partial \mathbf{R}_{0}^{\top}}{\partial \mathbf{r}_{0, i}} \mathbf{r}_{0}^{\prime}, \\
& \frac{\partial \varepsilon^{\prime}}{\partial \mathbf{r}_{0, i}}=\frac{\partial \mathbf{R}^{\prime \top}}{\partial \mathbf{r}_{0, i}} \mathbf{r}^{\prime}+\mathbf{R}^{\prime \top} \frac{\partial \mathbf{r}^{\prime}}{\partial \mathbf{r}_{0, i}}+\mathbf{R}^{\top} \frac{\partial \mathbf{r}^{\prime \prime}}{\partial \mathbf{r}_{0, i}}-\frac{\partial \mathbf{R}_{0}^{\prime \top}}{\partial \mathbf{r}_{0, i}} \mathbf{r}_{0}^{\prime}-\mathbf{R}_{0}^{\prime \top} \frac{\partial \mathbf{r}_{0}^{\prime}}{\partial \mathbf{r}_{0, i}}-\frac{\partial \mathbf{R}_{0}^{\top}}{\partial \mathbf{r}_{0, i}} \mathbf{r}_{0}^{\prime \prime}-\mathbf{R}_{0}^{\top} \frac{\partial \mathbf{r}_{0}^{\prime \prime}}{\partial \mathbf{r}_{0, i}} \\
& \frac{\partial \boldsymbol{\kappa}}{\partial \mathbf{r}_{0, i}}=\left(\begin{array}{l}
\mathbf{g}_{3}^{\top} \frac{\partial \mathbf{g}_{2}^{\prime}}{\partial \mathbf{r}_{0, i}} \\
\mathbf{g}_{1}^{\top} \frac{\partial \mathbf{g}_{3}^{\prime}}{\partial \mathbf{r}_{0, i}} \\
\mathbf{g}_{2}^{\top} \frac{\partial \mathbf{g}_{1}^{\prime}}{\partial \mathbf{r}_{0, i}}
\end{array}\right)-\left(\begin{array}{l}
\mathbf{g}_{0,3}^{\top} \frac{\partial \mathbf{g}_{0,2}^{\prime}}{\partial \mathbf{r}_{0, i}}+\mathbf{g}_{0,2}^{\prime \top} \frac{\partial \mathbf{g}_{0,3}}{\partial \mathbf{r}_{0, i}} \\
\mathbf{g}_{0,1}^{\top} \frac{\partial \mathbf{g}_{0,3}^{\prime}}{\partial \mathbf{r}_{0, i}}+\mathbf{g}_{0,3}^{\prime \top} \frac{\partial \mathbf{g}_{0,1}}{\partial \mathbf{r}_{0, i}} \\
\mathbf{g}_{0,2}^{\top} \frac{\partial \mathbf{g}_{0,1}^{\prime}}{\partial \mathbf{r}_{0, i}}+\mathbf{g}_{0,1}^{\prime \top} \frac{\partial \mathbf{g}_{0,2}}{\partial \mathbf{r}_{0, i}}
\end{array}\right) \\
& \frac{\partial \boldsymbol{\kappa}^{\prime}}{\partial \mathbf{r}_{0, i}}=\left(\begin{array}{l}
\mathbf{g}_{3}^{\top} \frac{\partial \mathbf{g}_{2}^{\prime \prime}}{\partial \mathbf{r}_{0, i}}+\mathbf{g}_{2}^{\prime \top} \frac{\partial \mathbf{g}_{3}^{\prime}}{\partial \mathbf{r}_{0, i}}+\mathbf{g}_{3}^{\prime \top} \frac{\partial \mathbf{g}_{2}^{\prime}}{\partial \mathbf{r}_{0, i}} \\
\mathbf{g}_{1}^{\top} \frac{\partial \mathbf{g}_{3}^{\prime \prime}}{\partial \mathbf{r}_{0, i}}+\mathbf{g}_{3}^{\prime \top} \frac{\partial \mathbf{g}_{1}^{\prime}}{\partial \mathbf{r}_{0, i}}+\mathbf{g}_{1}^{\prime \top} \frac{\partial \mathbf{g}_{3}^{\prime}}{\partial \mathbf{r}_{0, i}} \\
\mathbf{g}_{2}^{\top} \frac{\partial \mathbf{g}_{1}^{\prime \prime}}{\partial \mathbf{r}_{0, i}}+\mathbf{g}_{1}^{\prime \top} \frac{\partial \mathbf{g}_{2}^{\prime}}{\partial \mathbf{r}_{0, i}}+\mathbf{g}_{2}^{\prime \top} \frac{\partial \mathbf{g}_{1}^{\prime}}{\partial \mathbf{r}_{0, i}}
\end{array}\right)-\left(\begin{array}{l}
\mathbf{g}_{0,2}^{\prime \prime \top} \frac{\partial \mathbf{g}_{0,3}}{\partial \mathbf{r}_{0, i}}+\mathbf{g}_{0,3}^{\top} \frac{\partial \mathbf{g}_{0,2}^{\prime \prime}}{\partial \mathbf{r}_{0, i}}+\mathbf{g}_{0,2}^{\prime \top} \frac{\partial \mathbf{g}_{0,3}^{\prime}}{\partial \mathbf{r}_{0, i}}+\mathbf{g}_{0,3}^{\prime \top} \frac{\partial \mathbf{g}_{0,2}^{\prime}}{\partial \mathbf{r}_{0, i}} \\
\mathbf{g}_{0,3}^{\prime \prime \top} \frac{\partial \mathbf{g}_{0,1}}{\partial \mathbf{r}_{0, i}}+\mathbf{g}_{0,1}^{\top} \frac{\partial \mathbf{g}_{0,3}^{\prime \prime}}{\partial \mathbf{r}_{0, i}}+\mathbf{g}_{0,3}^{\prime \top} \frac{\partial \mathbf{g}_{0,1}^{\prime}}{\partial \mathbf{r}_{0, i}}+\mathbf{g}_{0,1}^{\prime \top} \frac{\partial \mathbf{g}_{0,3}^{\prime}}{\partial \mathbf{r}_{0, i}} \\
\mathbf{g}_{0,1}^{\prime \prime \top} \frac{\partial \mathbf{g}_{0,2}}{\partial \mathbf{r}_{0, i}}+\mathbf{g}_{0,2}^{\top} \frac{\partial \mathbf{g}_{0,1}^{\prime \prime}}{\partial \mathbf{r}_{0, i}}+\mathbf{g}_{0,1}^{\prime \top} \frac{\partial \mathbf{g}_{0,2}^{\prime}}{\partial \mathbf{r}_{0, i}}+\mathbf{g}_{0,2}^{\prime \top} \frac{\partial \mathbf{g}_{0,1}^{\prime}}{\partial \mathbf{r}_{0, i}}
\end{array}\right)
\end{aligned}
$$

Using the sensitivity expression for the strains, we can now also derive the sensitivities of the translational 
and rotational stress resultants, see (8):

$$
\begin{aligned}
\frac{\partial \boldsymbol{\sigma}}{\partial \mathbf{r}_{0, i}} & =\mathbf{A} \frac{d \boldsymbol{\varepsilon}}{d \mathbf{r}_{0, i}}+\mathbf{B} \frac{\partial \boldsymbol{\kappa}}{\partial \mathbf{r}_{0, i}}, \\
\frac{\partial \boldsymbol{\sigma}^{\prime}}{\partial \mathbf{r}_{0, i}} & =\frac{\partial \mathbf{A}^{\prime}}{\partial \mathbf{r}_{0, i}} \boldsymbol{\varepsilon}+\mathbf{A}^{\prime} \frac{\partial \boldsymbol{\varepsilon}}{\partial \mathbf{r}_{0, i}}+\mathbf{A} \frac{\partial \boldsymbol{\varepsilon}^{\prime}}{\partial \mathbf{r}_{0, i}}+\frac{\partial \mathbf{B}^{\prime}}{\partial \mathbf{r}_{0, i}} \boldsymbol{\kappa}+\mathbf{B}^{\prime} \frac{\partial \boldsymbol{\kappa}}{\partial \mathbf{r}_{0, i}}+\mathbf{B} \frac{\partial \boldsymbol{\kappa}^{\prime}}{\partial \mathbf{r}_{0, i}} \\
\frac{\partial \boldsymbol{\chi}}{\partial \mathbf{r}_{0, i}} & =\mathbf{B}^{\top} \frac{\partial \boldsymbol{\varepsilon}}{\partial \mathbf{r}_{0, i}}+\mathbf{C} \frac{\partial \boldsymbol{\kappa}}{\partial \mathbf{r}_{0, i}}, \\
\frac{\partial \boldsymbol{\chi}^{\prime}}{\partial \mathbf{r}_{0, i}} & =\frac{\partial \mathbf{B}^{\prime \top}}{\partial \mathbf{r}_{0, i}} \boldsymbol{\varepsilon}+\mathbf{B}^{\prime \top} \frac{\partial \boldsymbol{\varepsilon}}{\partial \mathbf{r}_{0, i}}+\mathbf{B}^{\top} \frac{\partial \boldsymbol{\varepsilon}^{\prime}}{\partial \mathbf{r}_{0, i}}+\frac{\partial \mathbf{C}^{\prime}}{\partial \mathbf{r}_{0, i}} \boldsymbol{\kappa}+\mathbf{C}^{\prime} \frac{\partial \boldsymbol{\kappa}}{\partial \mathbf{r}_{0, i}}+\mathbf{C} \frac{\partial \boldsymbol{\kappa}^{\prime}}{\partial \mathbf{r}_{0, i}}
\end{aligned}
$$

Next, the sensitivities of the internal forces and moments, see $[10]$, can be computed:

$$
\begin{aligned}
\frac{\partial \mathbf{n}}{d \mathbf{r}_{0, i}} & =\mathbf{R} \frac{\partial \boldsymbol{\sigma}}{\partial \mathbf{r}_{0, i}}, \\
\frac{\partial \mathbf{n}^{\prime}}{d \mathbf{r}_{0, i}} & =\frac{\partial \mathbf{R}^{\prime}}{\partial \mathbf{r}_{0, i}} \boldsymbol{\sigma}+\mathbf{R}^{\prime} \frac{\partial \boldsymbol{\sigma}}{\partial \mathbf{r}_{0, i}}+\mathbf{R} \frac{\partial \boldsymbol{\sigma}^{\prime}}{\partial \mathbf{r}_{0, i}}, \\
\frac{\partial \mathbf{m}}{d \mathbf{r}_{0, i}} & =\mathbf{R} \frac{\partial \boldsymbol{\chi}}{\partial \mathbf{r}_{0, i}}, \\
\frac{\partial \mathbf{m}^{\prime}}{d \mathbf{r}_{0, i}} & =\frac{\partial \mathbf{R}^{\prime}}{\partial \mathbf{r}_{0, i}} \boldsymbol{\chi}+\mathbf{R}^{\prime} \frac{\partial \boldsymbol{\chi}}{\partial \mathbf{r}_{0, i}}+\mathbf{R} \frac{\partial \boldsymbol{\chi}^{\prime}}{\partial \mathbf{r}_{0, i}}
\end{aligned}
$$

Finally, the sensitivities of the equilibrium equations of linear and angular momentum, see (11) and (18), are given by:

$$
\begin{aligned}
\frac{\partial \mathbf{f}_{\mathbf{n}}}{\partial \mathbf{r}_{0, i}} & =\frac{\partial \mathbf{n}^{\prime}}{\partial \mathbf{r}_{0, i}}, \\
\frac{\partial \mathbf{f}_{\mathbf{m}}}{\partial \mathbf{r}_{0, i}} & =\frac{\partial \mathbf{m}^{\prime}}{\partial \mathbf{r}_{0, i}}+\frac{\partial \mathbf{r}^{\prime}}{\partial \mathbf{r}_{0, i}} \times \mathbf{n}+\mathbf{r}^{\prime} \times \frac{\partial \mathbf{n}}{\partial \mathbf{r}_{0, i}} .
\end{aligned}
$$

By evaluating these expressions for all collocation points $\tau_{j},(j=1, \ldots, n)$ and control points $\mathbf{r}_{0, i},(i=$ $\left.1, \ldots, n_{0}\right)$, the sensitivity matrix $\mathbf{K}_{u}=\frac{d \overrightarrow{\mathbf{f}}}{d \overrightarrow{\mathbf{u}}}$ can be assembled.

Furthermore, for rod structures with $n_{I}$ rods being connected at an interface, the combined nonlinear force residual vector has to be adjusted to ensure equilibrium of linear and angular momentum, as well as continuity of deformed centerline positions and change of rotation. Consequently, the sensitivities of the modified rows corresponding to the collocation point of the first rod, see (21), become:

$$
\begin{gathered}
\frac{\partial \mathbf{f}_{\mathrm{n}}^{(1)}}{\partial \mathbf{r}_{0, i}} \leftarrow \sum_{k=1}^{n_{I}} I_{k} \frac{\partial \mathbf{f}_{\mathrm{n}}^{(k)}}{\partial \mathbf{r}_{0, i}} \\
\frac{\partial \mathbf{f}_{\mathrm{m}}^{(1)}}{\partial \mathbf{r}_{0, i}} \leftarrow \sum_{k=1}^{n_{I}} I_{k} \frac{\partial \mathbf{f}_{\mathrm{m}}^{(k)}}{\partial \mathbf{r}_{0, i}}
\end{gathered}
$$

and for the rows corresponding to interfacing rods $k=2, \ldots, n_{I}$, see 22 :

$$
\begin{gathered}
\frac{\partial \mathbf{f}_{\mathbf{n}}^{(k)}}{\partial \mathbf{r}_{0, i}} \leftarrow \frac{\partial}{\partial \mathbf{r}_{0, i}}\left(\mathbf{r}^{(1)}-\mathbf{r}^{(k)}\right)=\mathbf{0}, \\
\frac{\partial \mathbf{f}_{\mathbf{m}}^{(k)}}{\partial \mathbf{r}_{0, i}} \leftarrow \mathbf{q}^{(1)} \frac{\partial \overline{\mathbf{q}}_{0}^{(1)}}{\partial \mathbf{r}_{0, i}}-\mathbf{q}^{(k)} \frac{\partial \overline{\mathbf{q}}_{0}^{(k)}}{\partial \mathbf{r}_{0, i}}
\end{gathered}
$$




\subsubsection{Sensitivities of initial cross-section orientations}

In the derivation of the sensitivity matrix in the preceding Section 4.2 .2 we have so far not discussed that the initial cross-section orientations are typically determined from the initial centerline curve $\mathbf{r}_{0}$, see Remark 1 . This means that the control points of the quaternion curve $\mathbf{q}_{0}$ actually depend on the centerline curve and its control points, i.e., $\mathbf{q}_{0, j} \equiv \mathbf{q}_{0, j}\left(\mathbf{r}_{0, i}\right)$. Consequently, all quantities derived from $\mathbf{q}_{0}$, such as $\mathbf{R}_{0}, \boldsymbol{\kappa}_{0}$ and their arc-length derivatives, also implicitly depend on the design variables $\mathbf{r}_{0, i}$. Thus, in (31) expressions such as the sensitivities of $\mathbf{R}_{0}$ and its columns $\mathbf{g}_{0, k}$, or the derivatives of $\mathbf{q}_{0}$ in (36) are not necessarily 0 and for the arc-length derivatives it holds, e.g.,

$$
\frac{\partial \mathbf{R}_{0}}{\partial \mathbf{r}_{0, i}}=\sum_{j} \frac{\partial \mathbf{R}_{0}}{\partial \mathbf{q}_{0, j}} \frac{\partial \mathbf{q}_{0, j}}{\partial \mathbf{r}_{0, i}}, \quad \frac{\partial \mathbf{R}_{0}^{\prime}}{\partial \mathbf{r}_{0, i}} \equiv \frac{\partial \mathbf{R}_{0}^{\prime}}{\partial \mathbf{r}_{0, i}}+\sum_{j} \frac{\partial \mathbf{R}_{0}^{\prime}}{\partial \mathbf{q}_{0, j}} \frac{\partial \mathbf{q}_{0, j}}{\partial \mathbf{r}_{0, i}}, \quad \frac{\partial \mathbf{R}_{0}^{\prime \prime}}{\partial \mathbf{r}_{0, i}} \equiv \frac{\partial \mathbf{R}_{0}^{\prime \prime}}{\partial \mathbf{r}_{0, i}}+\sum_{j} \frac{\partial \mathbf{R}_{0}^{\prime \prime}}{\partial \mathbf{q}_{0, j}} \frac{\partial \mathbf{q}_{0, j}}{\partial \mathbf{r}_{0, i}} .
$$

However, obtaining the sensitivities of the initial quaternions $\frac{\partial \mathbf{q}_{0, j}}{\partial \mathbf{r}_{0, i}}$ is complex and in the case of the Bishop frame, which is only defined implicitly, see (7), they have to be determined numerically. Thus, one would either have to:

i) determine the quaternion sensitivities $\frac{\partial \mathbf{q}_{0, j}}{\partial \mathbf{r}_{0, i}}$ numerically by a finite difference scheme that re-computes the frame for small variations of the curve, or

ii) use an adjoint approach to determine the quaternion sensitivities $\frac{\partial \mathbf{q}_{0, j}}{\partial \mathbf{r}_{0, i}}$ implicitly from the definition of the Bishop frame, see Sect. 4.2 .3 , or

iii) determine the whole $\mathbf{K}_{u}$ matrix numerically by a finite difference scheme, see option a) above, or

iv) ignore the dependence of the frame on the centerline by setting the quaternion sensitivities to 0 and thus obtain an inconsistent analytical $\mathbf{K}_{u}$.

Since the first option is computationally expensive and inefficient, we will further discuss and validate only the latter three approaches to compute the gradient and sensitivities in the numerical examples following in Section 5.1

\subsubsection{Implicit sensitivities of initial cross-section orientations through Bishop frame}

As outlined above in Sect. 2 and Remark 1. the cross-section orientations $\mathbf{R}_{0}(s)$ and the quaternion control points $\mathbf{q}_{0, i}$ used to define them are basically arbitrary (or user-defined). Typically, they are initialized dependent on $\mathbf{r}_{0}$ and its derivatives, thus they are dependent on $\overrightarrow{\mathbf{u}}$. Here, we use the so-called Bishop frame, which is defined implicitly through the conditions, see (7):

$$
\mathbf{g}_{0,3}=\mathbf{r}_{0}^{\prime} \quad \wedge \quad \mathbf{k}_{0,3}=0 \quad \forall s \in[0, L] .
$$

To compute the quaternion control points $\overrightarrow{\mathbf{q}}_{0}=\left\{\mathbf{q}_{0, i}\right\}_{i=1, \ldots, n}$ numerically, we reformulate those conditions and evaluate them at the collocation points $\tau_{i}, i=1, \ldots, n$ :

$$
\overrightarrow{\mathbf{h}}_{\mathbf{q}}\left(\overrightarrow{\mathbf{q}}_{0}\right)=\mathbf{0}: \quad \mathbf{h}_{\mathbf{q}}\left(\tau_{1}\right):=\left(\begin{array}{c}
\mathbf{g}_{0,1}^{\top} \mathbf{r}_{0}^{\prime} \\
\mathbf{g}_{0,2}^{\top} \mathbf{r}_{0}^{\prime} \\
\mathbf{g}_{0,1}^{\top} \mathbf{h}_{0} \\
\mathbf{q}^{\top} \mathbf{q}-1
\end{array}\right)=\mathbf{0}, \quad \mathbf{h}_{\mathbf{q}}\left(\tau_{i}\right):=\left(\begin{array}{c}
\mathbf{g}_{0,1}^{\top} \mathbf{r}_{0}^{\prime} \\
\mathbf{g}_{0,2}^{\top} \mathbf{r}_{0}^{\prime} \\
\mathbf{g}_{0,2}^{\top} \mathbf{g}_{0,1}^{\prime} \\
\mathbf{q}^{\top} \mathbf{q}-1
\end{array}\right)=\mathbf{0}
$$

Here, the ambiguity of the rotation of the frame $\mathbf{R}_{0}$ around $\mathbf{r}_{0}^{\prime}$ is resolved by the boundary condition for $\tau_{1}$, where $\mathbf{h}_{0} \in \mathbb{R}^{3}$ is an arbitrary, user-defined normalized vector which must not be collinear to $\mathbf{r}_{0}^{\prime}$. Altogether, this yields a system of $4 n$ nonlinear equations that implicitly defines the $4 n$ initial quaternion control points, $\overrightarrow{\mathbf{h}}_{\mathbf{q}}\left(\overrightarrow{\mathbf{q}}_{0}\right)=\mathbf{0}$, which is solved using a Newton's method.

Now, in the context of the design optimization problem (24), we can view (39) as another implicitly defined constraint. Following the argumentation for $\overrightarrow{\mathbf{f}}(\overrightarrow{\mathbf{x}} ; \overrightarrow{\mathbf{u}})=\mathbf{0}$, see Sect. 4.2 also $\overrightarrow{\mathbf{h}}_{\mathrm{q}}\left(\overrightarrow{\mathbf{q}}_{0} ; \overrightarrow{\mathbf{u}}\right)=\mathbf{0}$ needs to 
be satisfied for every design $\overrightarrow{\mathbf{u}}$ and is thus stationary, i.e.:

$$
\mathbf{0} \equiv \frac{D \overrightarrow{\mathbf{h}}_{\mathrm{q}}}{D \overrightarrow{\mathbf{u}}}=\frac{d \overrightarrow{\mathbf{h}}_{\mathrm{q}}}{d \overrightarrow{\mathbf{u}}}+\frac{d \overrightarrow{\mathbf{h}}_{\mathrm{q}}}{d \overrightarrow{\mathbf{q}}_{0}} \frac{d \overrightarrow{\mathbf{q}}_{0}}{d \overrightarrow{\mathbf{u}}} \Rightarrow \frac{d \overrightarrow{\mathbf{q}}_{0}}{d \overrightarrow{\mathbf{u}}}=-\left(\frac{d \overrightarrow{\mathbf{h}}_{\mathrm{q}}}{d \overrightarrow{\mathbf{q}}_{0}}\right)^{-1} \frac{d \overrightarrow{\mathbf{h}}_{\mathrm{q}}}{d \overrightarrow{\mathbf{u}}}
$$

Thus, we can compute the sensitivities of the initial quaternions $\frac{d \overrightarrow{\mathbf{q}}_{0}}{d \overrightarrow{\mathbf{u}}}=\left(\frac{\partial \mathbf{q}_{0, j}}{\partial \mathbf{r}_{0, i}}\right)_{j i}$ by solving the linear system. Therefore, the analytical derivatives $\frac{d \overrightarrow{\mathbf{h}}_{\mathrm{q}}}{d \overrightarrow{\mathbf{q}}_{0}}$ and $\frac{d \overrightarrow{\mathbf{h}}_{\mathrm{q}}}{d \overrightarrow{\mathbf{u}}}$ are given below for $\tau_{1}$ and $\tau_{i}, i=2, \ldots, n$ :

$$
\begin{aligned}
& \frac{\partial \mathbf{h}_{\mathbf{q}}}{\partial \mathbf{q}_{0, j}}\left(\tau_{1}\right)=\left(\begin{array}{c}
\frac{\partial \mathbf{g}_{0,1}^{\top}}{\partial \mathbf{q}_{0, j}} \mathbf{r}_{0}^{\prime} \\
\frac{\partial \mathbf{g}_{0,2}^{\top}}{\partial \mathbf{q}_{0, j}} \mathbf{r}_{0}^{\prime} \\
\frac{\partial \mathbf{g}_{0,1}^{\top}}{\partial \mathbf{q}_{0, j}} \mathbf{h}_{0} \\
2 N_{j} \mathbf{q}_{0}^{\top}
\end{array}\right), \quad \frac{\partial \mathbf{h}_{\mathbf{q}}}{\partial \mathbf{q}_{0, j}}\left(\tau_{i}\right)=\left(\begin{array}{c}
\frac{\partial \mathbf{g}_{0,1}^{\top}}{\partial \mathbf{q}_{0, j}} \mathbf{r}_{0}^{\prime} \\
\frac{\partial \mathbf{g}_{0,2}^{\prime}}{\partial \mathbf{q}_{0, j}} \mathbf{r}_{0}^{\prime} \\
\frac{\partial \mathbf{g}_{0,2}^{\top}}{\partial \mathbf{q}_{0, j}} \mathbf{g}_{0,1}+\frac{\partial \mathbf{g}_{0,1}^{\top}}{\partial \mathbf{q}_{0, j}} \mathbf{g}_{0,1} \\
2 N_{j} \mathbf{q}_{0}^{\top}
\end{array}\right), \\
& \frac{\partial \mathbf{h}_{\mathbf{q}}}{\partial \mathbf{r}_{0, k}}\left(\tau_{1}\right)=\left(\begin{array}{c}
\mathbf{g}_{0,1}^{\top} N_{0, k}^{\prime} \\
\mathbf{g}_{0,2}^{\top} N_{0, k}^{\prime} \\
\mathbf{0}^{\top} \\
\mathbf{0}^{\top}
\end{array}\right), \quad \frac{\partial \mathbf{h}_{\mathbf{q}}}{\partial \mathbf{r}_{0, k}}\left(\tau_{i}\right)=\left(\begin{array}{c}
\mathbf{g}_{0,1}^{\top} N_{0, k}^{\prime} \\
\mathbf{g}_{0,2}^{\top} N_{0, k}^{\prime} \\
\mathbf{g}_{0,2}^{\top} \frac{\partial \mathbf{g}_{0,1}^{\prime}}{\partial \mathbf{r}_{0, k}} \\
\mathbf{0}^{\top}
\end{array}\right) .
\end{aligned}
$$

\subsection{Objective functions and constraints}

To complete the mathematical formulation of the nonlinear optimization problem (24), we need to define the scalar objective function $g: \mathbb{R}^{n_{u}} \times \mathbb{R}^{n_{x}} \rightarrow \mathbb{R}$ and (optionally) also the nonlinear equality constraints $\overrightarrow{\mathbf{h}}: \mathbb{R}^{n_{u}} \rightarrow \mathbb{R}^{n_{h}}$. In the following, we introduce the definitions of the types of objective functions and constraints that we use to optimize the mechanical performance of a $3 \mathrm{D}$ beam or beam structure.

\subsubsection{Shape matching objective}

We investigate so-called shape matching problems, where the shape of the beam in the deformed configuration given by $\mathbf{r}, \mathbf{q}$ or $\boldsymbol{\kappa}$ should be as close to possible to a desired target configuration given by $\mathbf{r}_{t}$, $\mathbf{q}_{t}$ or $\boldsymbol{\kappa}_{t}$. Thus, the deviation between those two configurations should be minimized. Here, we measure the deviation of the deformed configuration from the target shape in terms of the deviations of centerline curve, quaternions or curvatures at the collocation points:

$$
g(\overrightarrow{\mathbf{u}} ; \overrightarrow{\mathbf{x}}(\overrightarrow{\mathbf{u}}))=\frac{C_{r}}{2} \sum_{j=1}^{n}\left\|\mathbf{r}\left(\tau_{j}\right)-\mathbf{r}_{t}\left(\tau_{j}\right)\right\|^{2}+\frac{C_{q}}{2} \sum_{j=1}^{n}\left\|\mathbf{q}\left(\tau_{j}\right)-\mathbf{q}_{t}\left(\tau_{j}\right)\right\|^{2}+\frac{C_{k}}{2} \sum_{j=1}^{n}\left\|\boldsymbol{\kappa}\left(\tau_{j}\right)-\boldsymbol{\kappa}_{t}\left(\tau_{j}\right)\right\|^{2},
$$

where $C_{r}, C_{q}, C_{k} \geq 0$ serve as weights.

This definition of $g$ clearly depends explicitly on the state variables $\overrightarrow{\mathbf{x}}$, which depend implicitly on the design variables $\overrightarrow{\mathbf{u}}$, but also explicitly on $\overrightarrow{\mathbf{u}}$ if $C_{k}>0$, see (31). Thus, the sensitivities required to compute the gradient using the adjoint approach, see 25$]$, are:

$$
\begin{aligned}
\frac{\partial g}{\partial \mathbf{r}_{0, i}} & =C_{k} \sum_{j=1}^{n}\left(\boldsymbol{\kappa}\left(\tau_{j}\right)-\boldsymbol{\kappa}_{t}\left(\tau_{j}\right)\right)^{\top} \frac{\partial \boldsymbol{\kappa}\left(\tau_{j}\right)}{\partial \mathbf{r}_{0, i}}, \\
\frac{\partial g}{\partial \mathbf{r}_{i}} & =C_{r} \sum_{j=1}^{n}\left(\mathbf{r}\left(\tau_{j}\right)-\mathbf{r}_{t}\left(\tau_{j}\right)\right)^{\top} N_{i}\left(\tau_{j}\right), \\
\frac{\partial g}{\partial \mathbf{q}_{i}} & =C_{q} \sum_{j=1}^{n}\left(\mathbf{q}\left(\tau_{j}\right)-\mathbf{q}_{t}\left(\tau_{j}\right)\right)^{\top} N_{i}\left(\tau_{j}\right)+C_{k} \sum_{j=1}^{n}\left(\boldsymbol{\kappa}\left(\tau_{j}\right)-\boldsymbol{\kappa}_{t}\left(\tau_{j}\right)\right)^{\top} \frac{\partial \boldsymbol{\kappa}\left(\tau_{j}\right)}{\partial \mathbf{q}_{i}} .
\end{aligned}
$$




\subsubsection{End-point matching objectives}

As an alternative type of "shape"-matching objective functions, we demand only the end-points of a beam to match a given position $\mathbf{r}_{t}$, internal force $\mathbf{n}_{t}$ or internal moment value $\mathbf{m}_{t}$. Thus, the objective function only includes the evaluations at end-collocation points $\tau_{1}$ and $\tau_{n}$ :

$$
g(\overrightarrow{\mathbf{u}} ; \overrightarrow{\mathbf{x}}(\overrightarrow{\mathbf{u}}))=\frac{C_{r}}{2} \sum_{j=1, n}\left\|\mathbf{r}\left(\tau_{j}\right)-\mathbf{r}_{t, j}\right\|^{2}+\frac{C_{n}}{2} \sum_{j=1, n}\left\|\mathbf{n}\left(\tau_{j}\right)-\mathbf{n}_{t, j}\right\|^{2}+\frac{C_{m}}{2} \sum_{j=1, n}\left\|\mathbf{m}\left(\tau_{j}\right)-\mathbf{m}_{t, j}\right\|^{2},
$$

where $C_{r}, C_{n}, C_{m} \geq 0$ weigh the position, force and moment contributions. The sensitivities are:

$$
\begin{aligned}
\frac{\partial g}{\partial \mathbf{r}_{0, i}} & =C_{n} \sum_{j=1, n}\left(\mathbf{n}\left(\tau_{j}\right)-\mathbf{n}_{t, j}\right)^{\top} \frac{\partial \mathbf{n}\left(\tau_{j}\right)}{\partial \mathbf{r}_{0, i}}+C_{m} \sum_{j=1, n}\left(\mathbf{m}\left(\tau_{j}\right)-\mathbf{m}_{t, j}\right)^{\top} \frac{\partial \mathbf{m}\left(\tau_{j}\right)}{\partial \mathbf{r}_{0, i}}, \\
\frac{\partial g}{\partial \mathbf{r}_{i}} & =C_{r} \sum_{j=1, n}\left(\mathbf{r}\left(\tau_{j}\right)-\mathbf{r}_{t, j}\right)^{\top} N_{i}\left(\tau_{j}\right)+C_{n} \sum_{j=1, n}\left(\mathbf{n}\left(\tau_{j}\right)-\mathbf{n}_{t, j}\right)^{\top} \frac{\partial \mathbf{n}\left(\tau_{j}\right)}{\partial \mathbf{r}_{i}} \\
& +C_{m} \sum_{j=1, n}\left(\mathbf{m}\left(\tau_{j}\right)-\mathbf{m}_{t, j}\right)^{\top} \frac{\partial \mathbf{m}\left(\tau_{j}\right)}{\partial \mathbf{r}_{i}}, \\
\frac{\partial g}{\partial \mathbf{q}_{i}} & =C_{n} \sum_{j=1, n}\left(\mathbf{n}\left(\tau_{j}\right)-\mathbf{n}_{t, j}\right)^{\top} \frac{\partial \mathbf{n}\left(\tau_{j}\right)}{\partial \mathbf{q}_{i}}+C_{m} \sum_{j=1, n}\left(\mathbf{m}\left(\tau_{j}\right)-\mathbf{m}_{t, j}\right)^{\top} \frac{\partial \mathbf{m}\left(\tau_{j}\right)}{\partial \mathbf{q}_{i}} .
\end{aligned}
$$

\subsubsection{Length and volume constraints}

A commonly used type of constraints in mechanical design optimization problems are volume constraints, e.g., to restrict the amount of material required to fabricate the beam. In the context of beam structures, one could restrict both the length $L_{0}$ or the volume $V_{0}$ of the beam, which are defined as:

$$
L_{0}=\int_{0}^{1}\left\|\dot{\mathbf{r}}_{0}(\xi)\right\| d \xi, \quad V_{0}=\int_{0}^{1} A(\xi)\left\|\dot{\mathbf{r}}_{0}(\xi)\right\| d \xi .
$$

Since the definition of the length is a special case of the volume with $A \equiv 1$, we formulate the inequality constraints for the volume $V_{0}$ only:

- $\overrightarrow{\mathbf{h}}(\overrightarrow{\mathbf{u}})=C_{V}\left(V_{0}-V^{*}\right) \leq 0$, to allow a max. volume of $V^{*}$,

- $\overrightarrow{\mathbf{h}}(\overrightarrow{\mathbf{u}})=C_{V}\left(V^{*}-V_{0}\right) \leq 0$, to allow a min. volume of $V^{*}$, and

- $\overrightarrow{\mathbf{h}}(\overrightarrow{\mathbf{u}})=C_{V}\left(\begin{array}{l}V_{0}-V^{*}-\varepsilon \\ V^{*}-V_{0}-\varepsilon\end{array}\right) \leq \mathbf{0}$, to allow only a volume equal to $V^{*}$.

Here, and similarly in the following constraint definitions, we introduce a scaling factor $C_{V}>0$ to weigh the magnitudes of constraints and their gradients against each other and against the objective function, and a parameter $\varepsilon>0$ in order to allow small constraint violations, which is particularly important when two inequality constraints are used to formulate an equality constraint.

The gradients of the volume constraints can be computed using the sensitivities of the volume:

$$
\frac{\partial V_{0}}{\partial \mathbf{r}_{0, i}}=\int_{0}^{1} A \dot{N}_{0, i} \frac{\dot{\mathbf{r}}_{0}^{\top}}{\left\|\dot{\mathbf{r}}_{0}\right\|} d \xi
$$

For beam structures, length or volume constraints could either be applied per beam, or for the total, added length or volume of all members of the structure.

\subsubsection{Constraints on speed of parameterization}

A general issue with shape optimization of 3D beams is that not every 3D spline curve also defines a mechanically meaningful beam configuration. Thus, artifacts such as self-intersections, small loops and very uneven, non-linear parameterizations should be avoided. 
To address the last issue, we introduce constraints on the "speed of parameterization", i.e., the value of the Jacobian of the parameterization $J$, see (15). In isogeometric analysis, linear parameterizations are typically preferred due to better convergence properties, see e.g. [70, which means that it should hold that $J(\xi) \equiv L_{0}$. Thus, we introduce a constraint that penalizes the averaged deviations of $J$ at the collocation points $\tau_{j}$ :

$$
\overrightarrow{\mathbf{h}}(\overrightarrow{\mathbf{u}})=\frac{C_{J}}{2 n} \sum_{j=1}^{n}\left(\left(\frac{J\left(\tau_{j}\right)}{L_{0}}-1\right)^{2}-\varepsilon^{2}\right)
$$

with scaling factor $C_{J}>0$. The corresponding sensitivities are:

$$
\frac{\partial \overrightarrow{\mathbf{h}}}{\partial \mathbf{r}_{0, i}}=\frac{C_{J}}{n} \sum_{j=1}^{n}\left(\left(\frac{J\left(\tau_{j}\right)}{L_{0}}-1\right)\left(\frac{1}{L_{0}} \frac{\partial J\left(\tau_{j}\right)}{\partial \mathbf{r}_{0, i}}-\frac{J\left(\tau_{j}\right)}{L_{0}^{2}} \frac{\partial L_{0}}{\partial \mathbf{r}_{0, i}}\right)\right)
$$

\subsubsection{Directional constraints for end-points}

Similar to clamped boundary conditions, where both the centerline curve position and orientation are fixed, it can be desirable to restrict not only the movement or position of a rod end-point during optimization, which is simply done be excluding it from the design vector $\overrightarrow{\mathbf{u}}$, but also to prescribe the tangential direction of the centerline. This simply means that the derivative of the initial centerline curve $\mathbf{r}_{0}^{\prime}$ should be equal (or close to) a given target direction vector $\mathbf{t}_{t} \in \mathbb{R}^{3},\left\|\mathbf{t}_{t}\right\|=1$. Thus, a directional constraint can be formulated as:

$$
\overrightarrow{\mathbf{h}}(\overrightarrow{\mathbf{u}})=\frac{C_{T}}{2}\left(\left\|\mathbf{r}_{0}^{\prime}-\mathbf{t}_{t}\right\|^{2}-\varepsilon^{2}\right) \leq 0,
$$

with $C_{T}>0$. Its derivatives are:

$$
\frac{\partial \overrightarrow{\mathbf{h}}}{\partial \mathbf{r}_{0, i}}=C_{T}\left(\mathbf{r}_{0}^{\prime}-\mathbf{t}_{t}\right)^{\top} \frac{\partial \mathbf{r}_{0}^{\prime}}{\partial \mathbf{r}_{0, i}}=C_{T}\left(\mathbf{r}_{0}^{\prime}-\mathbf{t}_{t}\right)^{\top} N_{0, i}^{\prime}\left(\mathbf{I}-\mathbf{r}_{0}^{\prime} \mathbf{r}_{0}^{\prime \top}\right) .
$$

\subsubsection{Interface constraints for beam structures}

When dealing with beam structures, it can be important to restrict the angles at which the beams meet at an interface. For instance, one could either want to preserve the angles used in the initial design, e.g., to ensure that the 4 beams at an interface meet at $90^{\circ}$ angles, or just to avoid extensive overlap and ensure that all angles are larger than a minimum angle. For an interface with $n_{I}$ rods, we thus introduce nonlinear inequality constraints for $k=1, \ldots, n_{I}$ :

$$
\overrightarrow{\mathbf{h}}_{k}(\overrightarrow{\mathbf{u}})=\frac{C_{S}}{2}\left(\left(\alpha_{k}-\operatorname{acos}\left(\mathbf{r}_{0}^{\prime(k)^{\top}} \mathbf{r}_{0}^{\prime(k+1)}\right)\right)^{2}-\varepsilon^{2}\right) \leq 0,
$$

where $C_{S}>0, n_{I}+1 \equiv 1$ and $\alpha_{k}$ is either the original angle between rods $k$ and $k+1$ or a given minimum angle. Then, the sensitivities of the constraints are:

$$
\frac{\partial \overrightarrow{\mathbf{h}}_{k}}{\partial \mathbf{r}_{0, i}^{(k)}}=C_{S} \frac{N_{0, i}^{(k)}\left(\alpha_{k}-\operatorname{acos}\left(\mathbf{r}_{0}^{\prime(k)^{\top}} \mathbf{r}_{0}^{\prime(k+1)}\right)\right) \mathbf{r}_{0}^{\prime(k+1)^{\top}}}{\sqrt{1-\mathbf{r}_{0}^{\prime(k)^{\top}} \mathbf{r}_{0}^{\prime(k+1)}}} .
$$

Furthermore, when dealing with beam structures, it needs to be ensured that the $n_{I}$ end-points $\mathbf{r}_{0, i}^{(k)}$ that meet at an interface maintain continuity through the optimization. Thus, the gradients of the objective function and all constraints need to be equal for those design variables. This is achieved by simply averaging their contributions in the gradient vectors. 


\section{Numerical results}

With a series of numerical examples, we now validate the shape optimization approach for nonlinear 3D beams and show its potential for engineering applications.

\subsection{Validation of gradient implementations}

As discussed in Section 4.2 , several approaches can be pursued to evaluate the gradient of the objective function for gradient-based optimization. In the following, we validate and compare the performance of three different methods, which are:

(FD) computing the complete gradient $\frac{D g}{D \overrightarrow{\mathbf{u}}}$ numerically by a finite difference approach;

(SA) using the adjoint method with a semi-analytical, finite difference approximation of the sensitivity matrix $\mathbf{K}_{u}$

(IA) using the adjoint method with an incomplete/inconsistent analytical evaluation of the sensitivity matrix $\mathbf{K}_{u}$, ignoring the dependence of the initial frame and quaternions on the centerline curve; and

(FA) using the adjoint method with fully analytical evaluation of the sensitivity matrix $\mathbf{K}_{u}$, solving the additional adjoint problem for the dependence of the initial quaternions on the centerline curve.

\subsubsection{Gradients of straight beam}

As a first simple example, we optimize an initially straight cantilever beam with length $L=1 \mathrm{~m}$, circular cross-section with radius $r=0.025 \mathrm{~m}$, Young's modulus $E=10 \mathrm{MPa}$ and Poisson's ratio $\nu=0.5$. The beam is oriented along the $x$-axis and a moment $M=2 \mathrm{Nm}$ around the $y$-axis is applied on its free end. This leads to pure bending deformation in the $x z$-plane with constant curvature $\kappa_{t}=0.652 \mathrm{~m}^{-1}$ around the $y$-axis, see Fig. 4a, which defines the target configuration $\mathbf{r}_{t}$ for the shape matching objective function $\left(C_{r}=1, C_{q}=C_{k}=0\right)$. Then, the moment is reduced to $\frac{1}{1} M=0.5 \mathrm{Nm}$, which leads to deformation with a constant curvature of only $\kappa_{t}=\frac{1}{4} \kappa_{t}=0.163$, see Fig. 4 a and thus requires optimizing the initial shape of the beam to match the target deformed shape. No constraints are applied and, trivially, the expected outcome is that the beam should be initially curved with $\kappa_{0}=\frac{3}{4} \kappa_{t}=0.489$ such that the deformed curvature will be $\kappa=\frac{1}{4} \kappa_{t}$ and $\kappa_{0}+\kappa=\kappa_{t}$, see Fig. $4 \mathrm{~b}$.

The spline space of the simulation discretization $\left\{N_{i}\right\}$ is chosen as B-Splines with $p=8, \ell=16, n=24$, while the coarser space of design variables $\left\{N_{0, i}\right\}$ are B-Splines with $p_{0}=4, \ell_{0}=1, n_{0}=5$. Since the first control point is fixed, the optimization problem has a total of $n_{u}=3\left(n_{0}-1\right)=12$ design variables. Generally, we use the Bishop frame to initialize the cross-section orientations, which is in this $2 \mathrm{D}$ problem equivalent to the Darboux frame.

In Table 1 we compare the gradient vectors $\frac{D g}{D \overrightarrow{\mathbf{u}}}$ of the objective function when evaluated at the beginning of the optimization, i.e., when the beam is in a straight shape, using FD, SA, IA and FA methods. For the finite difference schemes in the FD and SA approaches, a perturbation $\epsilon=10^{-7}$ is used. No significant influence of the value of $\epsilon$ on the accuracy of the results was observed for $10^{-8} \leq \epsilon \leq 10^{-5}$.

The numerical values resulting from all four methods, FD, SA, IA, and FA, are in excellent agreement with each other, which validates our implementation. However, one exception can be noticed: the $z_{2}$-values of the SA and FA methods are far off.

Intuitively, all $x$ - and $z$-values should be positive, since the gradient points towards the ascend direction of the objective function and to decrease $g$ and obtain the expected shape with constant curvature, the control points should move in the $-x$ - and $-z$-directions. However, for the SA and FA methods, there is a general issue when clamped boundary conditions (BC) are used, i.e., when displacement and orientation are fixed. Using the finite difference scheme to compute $\mathbf{K}_{u}$, the sensitivities are calculated from the difference between the converged nonlinear force residual vector $\overrightarrow{\mathbf{f}}(\overrightarrow{\mathbf{x}}(\overrightarrow{\mathbf{u}}) ; \overrightarrow{\mathbf{u}})$ and the force vector $\overrightarrow{\mathbf{f}}(\overrightarrow{\mathbf{x}}(\overrightarrow{\mathbf{u}}) ; \overrightarrow{\mathbf{u}} \epsilon)$, which is evaluated using the perturbed design vector $\overrightarrow{\mathbf{u}}_{\epsilon}$. However, if the second control point moves such that $\mathbf{r}_{0}^{\prime}\left(\overrightarrow{\mathbf{u}}_{\epsilon}\right) \neq \mathbf{r}_{0}^{\prime}(\overrightarrow{\mathbf{u}})$, the boundary behavior of the deformed configuration $\overrightarrow{\mathbf{x}}(\overrightarrow{\mathbf{u}})$ used to evaluate the perturbed $\overrightarrow{\mathbf{f}}$ is not consistent with the initial configuration defined by $\overrightarrow{\mathbf{u}}_{\epsilon}$. In the current example, this means that if the 


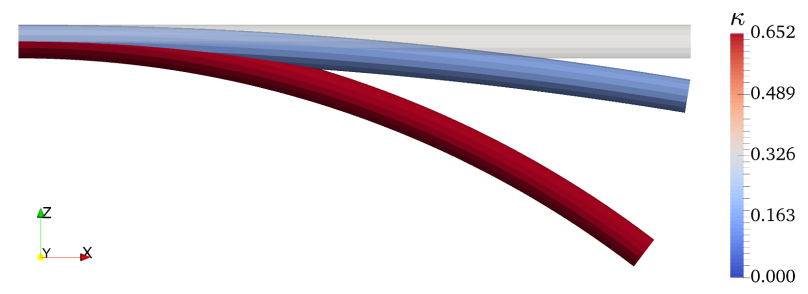

(a) Straight beam (gray) deforming under moment $M=2$ Nm (red) and reduced moment $\frac{1}{4} M$ (blue)

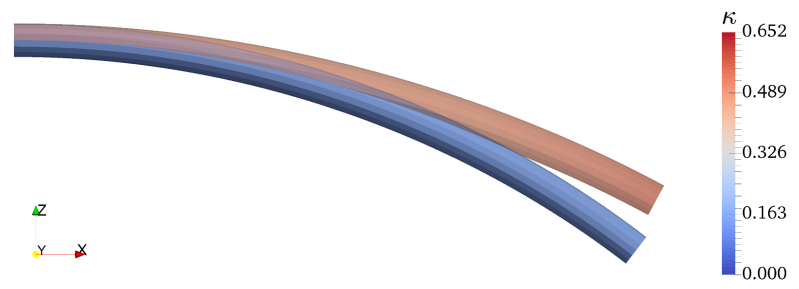

(b) Optimized, pre-curved beam (light red) deforming under reduced moment $\frac{1}{4} M$ (blue)
Table 1: Comparison of gradient vectors $\frac{D g}{D \overrightarrow{\mathbf{u}}}$ of straight beam resulting from finite difference $(\mathrm{FD})$, semianalytical adjoint (SA), incomplete analytical adjoint (IA), and fully analytical adjoint (FA \& FA*) methods

\begin{tabular}{rrrrrr}
\hline & FD & SA & IA & FA & FA $^{*}$ \\
\hline$x_{2}$ & 0.0062 & 0.0061 & 0.0062 & 0.0062 & 0.0062 \\
$y_{2}$ & 0.0000 & 0.0000 & 0.0000 & 0.0000 & 0.0000 \\
$z_{2}$ & 0.1014 & -7.1331 & 0.1014 & -7.1331 & 0.0996 \\
$x_{3}$ & 0.0164 & 0.0163 & 0.0164 & 0.0164 & 0.0164 \\
$y_{3}$ & 0.0000 & 0.0000 & 0.0000 & 0.0000 & 0.0000 \\
$z_{3}$ & 0.2254 & 0.2254 & 0.2254 & 0.2254 & 0.2254 \\
$x_{4}$ & 0.0397 & 0.0395 & 0.0396 & 0.0396 & 0.0396 \\
$y_{4}$ & 0.0000 & 0.0000 & 0.0000 & 0.0000 & 0.0000 \\
$z_{4}$ & 0.4567 & 0.4567 & 0.4567 & 0.4567 & 0.4567 \\
$x_{5}$ & 0.1352 & 0.1351 & 0.1352 & 0.1352 & 0.1352 \\
$y_{5}$ & 0.0000 & 0.0000 & 0.0000 & 0.0000 & 0.0000 \\
$z_{5}$ & 1.3281 & 1.3282 & 1.3281 & 1.3281 & 1.3281 \\
\hline
\end{tabular}

Figure 4: Validation example of straight beam

second control point moved in the positive $z$-direction, there would be a positive curvature $\kappa_{0}$ and thus the difference between the target and the deformed curvature became smaller, so the objective would decrease - thus, the gradient has a negative value. Likewise, this inconsistency in the boundary conditions appears when including $\frac{d \overrightarrow{\mathbf{q}}_{0}}{d \overrightarrow{\mathbf{u}}}$ in the FA method, since those terms consider the change of orientation at the clamped boundary. In the IA method, this issue does not appear, since $\frac{d \overrightarrow{\mathbf{q}}_{0}}{d \overrightarrow{\mathbf{u}}}$ is neglected. To resolve the problem for the FA method, we can exclude the equation and quaternion control point at the clamped boundary, i.e., here $\mathbf{h}_{\mathbf{q}}\left(\tau_{1}\right)$ and $\mathbf{q}_{0,1}$, from 40 , which yields the method denoted as FA*.

\subsubsection{Gradients of $3 D$ curved beam}

To further investigate the different gradient evaluation strategies, we move on to a more complex example with a 3-dimensionally curved beam. All parameters are the same as in Sect. 5.1.1, except that the initial control points of the beam are:

$$
\mathbf{r}_{0,1}=\left(\begin{array}{l}
0 \\
0 \\
0
\end{array}\right), \quad \mathbf{r}_{0,2}=\left(\begin{array}{c}
0.25 \\
0 \\
0
\end{array}\right), \quad \mathbf{r}_{0,3}=\left(\begin{array}{c}
0.5 \\
0.2 \\
0
\end{array}\right), \quad \mathbf{r}_{0,4}=\left(\begin{array}{c}
0.75 \\
0.2 \\
0.2
\end{array}\right), \quad \mathbf{r}_{0,5}=\left(\begin{array}{l}
1 \\
0 \\
0
\end{array}\right)
$$

and instead of the moment a force $\hat{\mathbf{n}}(L)=(0,-2,-3)^{\top} \mathrm{N}$ is applied to generate the target shape, and then decreased to $25 \%$ of its magnitude for the optimization, see Fig. 5 . Thus, also here a trivial optimal initial shape exists, which corresponds to the deformed configuration at $0.75 \hat{\mathbf{n}}$ applied load.

Again, the gradients of the first optimization step, i.e., when the shape corresponds to the curved shape defined by the control points given (54), are compared for FD, SA, IA and FA methods, see Tab. 2. Also here, good agreement of all four methods can be observed, which validates the implementation for this more complex, "arbitrarily" curved geometry. Even now, the error of the IA method compared to the other approaches is still small. The issue with clamped boundary conditions for the SA and FA methods, of course, also exists in this example and can simply be resolved by removing the first control point in the $\frac{d \overrightarrow{\mathbf{q}}_{0}}{d \overrightarrow{\mathbf{u}}}$ computation of the $\mathrm{FA}^{*}$ method. 


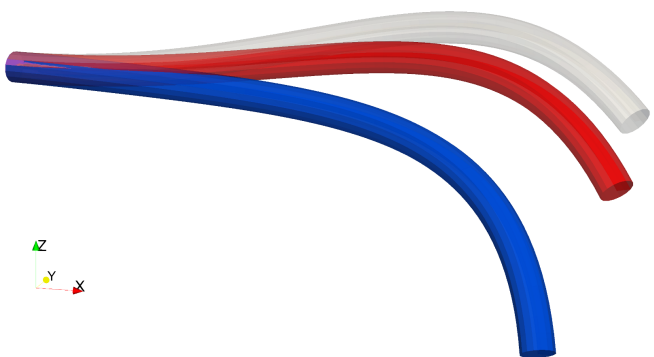

(a) $3 \mathrm{D}$ curved beam (gray) deforming under force $\hat{\mathbf{n}}=$ $(0,-2,-3)^{\top} \mathrm{N}$ (blue) and reduced force $\frac{1}{4} \hat{\mathbf{n}}$ (red)

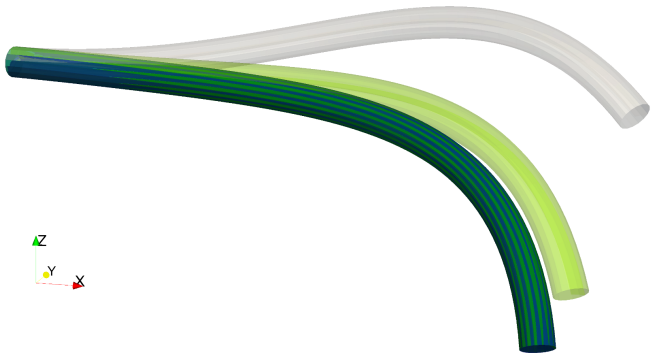

(b) Optimized 3D curved beam (light green) deforming under reduced force $\frac{1}{4} \hat{\mathbf{n}}$ (dark green) into target shape (blue)
Table 2: Comparison of gradient vectors $\frac{D g}{D \overrightarrow{\mathbf{u}}}$ of curved beam resulting from finite difference (FD), semi-analytical adjoint (SA), incomplete analytical adjoint (IA), and fully analytical adjoint (FA \& $\mathrm{FA}^{*}$ ) methods

\begin{tabular}{lrrrrr}
\hline \multicolumn{1}{c}{ FD } & SA & IA & FA & FA $^{*}$ \\
\hline$x_{2}$ & -0.0118 & -0.0118 & -0.0131 & -0.0118 & -0.0118 \\
$y_{2}$ & 0.0386 & -3.4808 & 0.0440 & -3.4808 & 0.0326 \\
$z_{2}$ & 0.1266 & -5.6185 & 0.1234 & -5.6185 & 0.1160 \\
$x_{3}$ & -0.0229 & -0.0229 & -0.0262 & -0.0229 & -0.0229 \\
$y_{3}$ & 0.0899 & 0.0899 & 0.1038 & 0.0899 & 0.0899 \\
$z_{3}$ & 0.2283 & 0.2283 & 0.2182 & 0.2283 & 0.2283 \\
$x_{4}$ & -0.0159 & -0.0162 & -0.0174 & -0.0159 & -0.0159 \\
$y_{4}$ & 0.2261 & 0.2261 & 0.2349 & 0.2260 & 0.2260 \\
$z_{4}$ & 0.3971 & 0.3971 & 0.3904 & 0.3970 & 0.3970 \\
$x_{5}$ & -0.0938 & -0.0941 & -0.0940 & -0.0939 & -0.0939 \\
$y_{5}$ & 0.6480 & 0.6479 & 0.6508 & 0.6480 & 0.6480 \\
$z_{5}$ & 0.9895 & 0.9895 & 0.9876 & 0.9895 & 0.9895 \\
\hline
\end{tabular}

Figure 5: Validation example of 3D curved beam

\subsubsection{Optimization}

Having compared and validated the different gradient evaluation strategies, we want to study their behavior when used within shape optimization algorithms. Thus, we now evaluate the performance of FD, SA, IA and FA methods within the above-define optimization problem of the 3D curved beam. Furthermore, we compare three nonlinear, gradient-based optimization algorithms from the NLopt library, namely Sequential Least-Squares Quadratic Programming (SLSQP), Method of Moving Asymptotes (MMA) and Preconditioned Truncated Newton (PTN), see [69, to validate the convergence behavior and speed of convergence, and determine the most suitable optimizer for beam optimization problems.

Figure 6 shows the convergence of the objective function value $g$ when using the three above-mentioned algorithms to solve the nonlinear optimization problem with gradient computation using the finite difference method (FD), see Fig. 6a. the semi-analytical adjoint method with finite difference sensitivities (SA) and the fully analytical adjoint method (FA), see Fig. 6b. the incomplete analytical adjoint method with inconsistent sensitivities (IA), see Fig. 6c and the fully analytical adjoint method with resolved clamped BC issue (FA*), see Fig. 6d. In all cases, max. 30 iterations are shown and relative and absolute termination tolerances for the change of objective function $g$ and design vector $\overrightarrow{\mathbf{u}}$ of $\epsilon=10^{-6}$ were used (though these might be interpreted differently by each optimizer [69]).

With both the IA method and the FA* method, all 3 optimizers exhibit fast convergence behavior, which suggests that the implementations are correct. Even the inconsistent sensitivities are sufficiently accurate to obtain a gradient that points in the correct direction and thus effectively drives the optimization towards the minimum, here 0 . The behavior using the FD gradient is very similar for both the SLSQP and MMA optimizers, but slightly worse for the PTN method. This probably means that the finite difference gradients do not provide sufficient accuracy to obtain a good approximation of the Hessian, which is what the PTN optimizer is doing when it plateaus and is repeatedly obtaining very similar values for $g$, since it is evaluating for very similar values of $\overrightarrow{\mathbf{u}}$.

The SA and FA methods, however, do not convergence towards 0 , which is here only shown for the SLSQP optimizers, since the above-mentioned issue with the boundary conditions persists throughout the optimization and the second control point thus moves in the wrong direction. However, when removing 


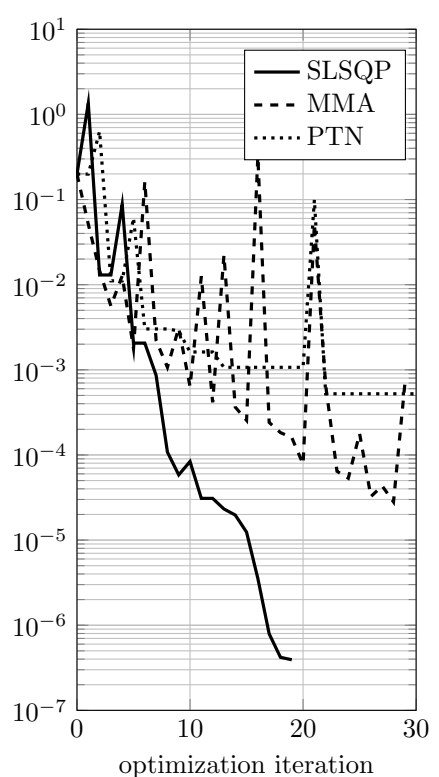

(a) FD gradient (FD)

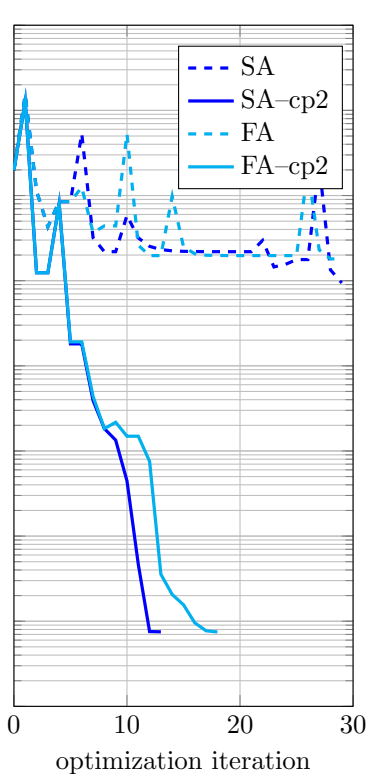

(b) semi-analytical (SA) \& fully-analytical adjoint (FA)

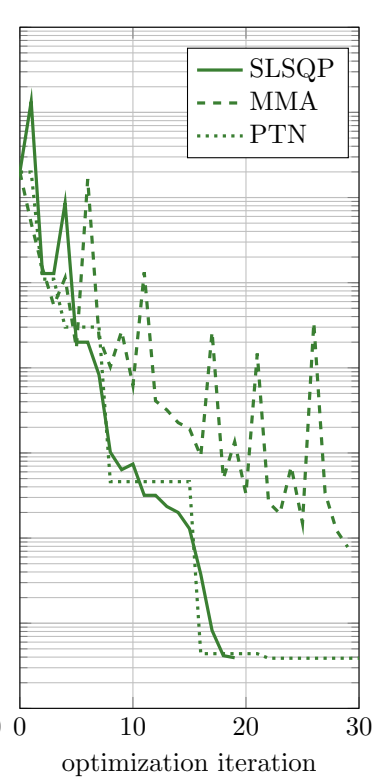

(c) incomplete analytical adjoint (IA)

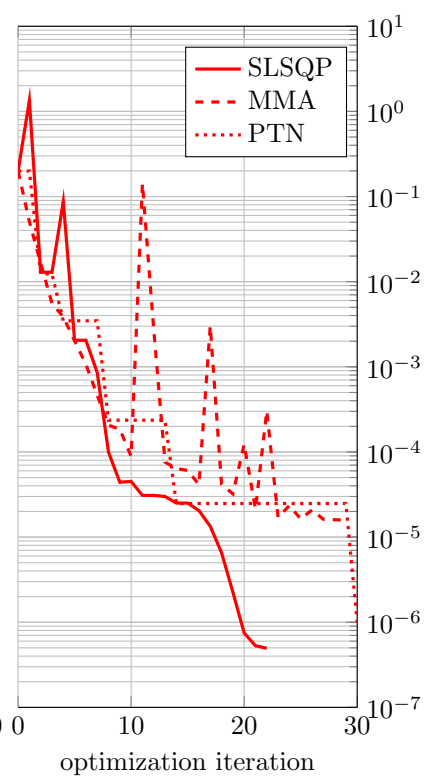

(d) fully analytical adjoint $\left(\mathrm{FA}^{*}\right)$

Figure 6: Convergence of objective function value during optimization of 3D curved beam with reduced force for FD, SA, IA and FA gradient evaluation methods and SLSQP, MMA and PTN optimization algorithms

the second control point $\mathbf{r}_{0,2}$ as a design variable, optimal convergence similar to the other methods can be observed, see curves $S A-c p 2$ and $F A-c p 2$ in Fig. 6b. Note that during the first iterations, SA and FA methods behave the same way and exhibit the same objective function values, which is to be expected as the sensitivity matrices should be equal. However, at some point they diverge from each other due to numerical inaccuracies.

We have also verified these general behaviors for refined spline spaces $\left\{N_{0, i}\right\}$ for the parameterizations of the design variables (along with refined spline spaces $\left\{N_{i}\right\}$ for the discretizations of the simulations variables, which are required to maintain the accuracy of the simulations). Furthermore, we have obtained similar, very good convergence behavior when rectangular instead of circular cross-sections are used. This is an important observation, as any rotation of the cross-section, i.e. of $\mathbf{g}_{0,1}$ and $\mathbf{g}_{0,2}$, around $\mathbf{g}_{0,3}=\mathbf{r}_{0}^{\prime}$ does not influence the mechanics when a circular cross-section is used, whereas it does for rectangular cross-sections.

In summary, this means that not only the fully analytical sensitivities (in the FA* method with resolved clamped BC issue), but also the inconsistent sensitivities in the IA method yield sufficiently accurate approaches to obtain the gradient of the objective function and efficiently perform shape optimization of nonlinear 3D beams. In fact, the IA method seems to be advantageous, as it does not exhibit the boundary conditions issue. The SA and FA methods also work effectively when the adjacent control point to the end is removed from the design space for clamped boundary conditions.

Using the FD approach is not feasible in larger scale design optimization problems, since the cost of directly computing the gradient from finite differences involves a nonlinear solve for every design variable. Thus, we will mainly use the computationally most efficient, analytical IA and FA methods in conjunction with the SLSQP and MMA optimizers in the our numerical examples and applications. In the following, we will drop the $\mathrm{FA}^{*}$ notation and use this variant synonymous with FA whenever clamped BC are used.

\subsection{Optimization of $3 D$ beam applications}

Having validated the implementation of the isogeometric shape optimization approach, the gradient evaluation strategies and nonlinear optimization algorithms, we now present two applications for optimization of $3 \mathrm{D}$ beams. 


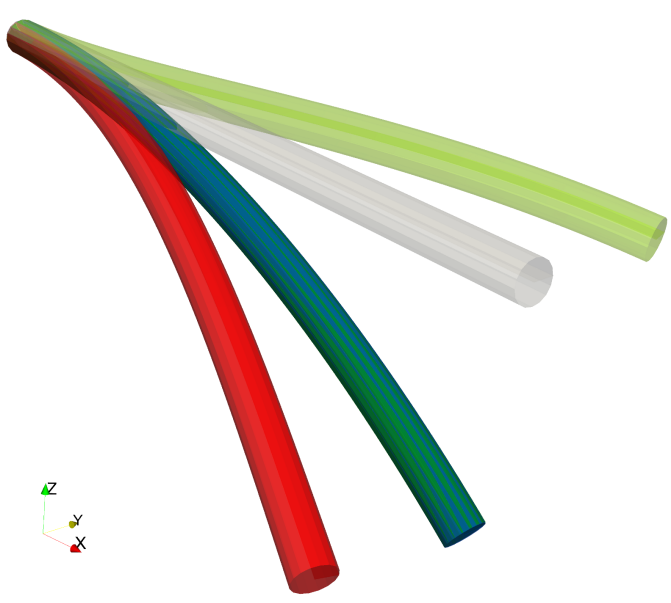

(a) $3 \mathrm{D}$ view

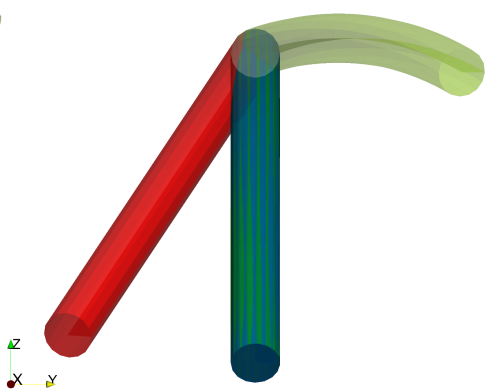

(b) $y z$-plane view

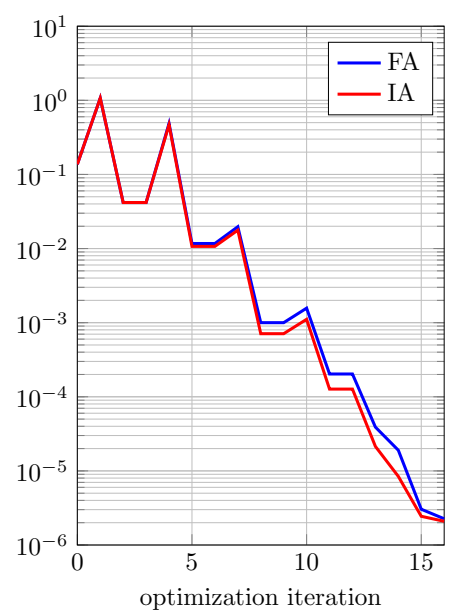

(c) convergence of objective

Figure 7: Optimization of beam to achieve constant curvature when an end-point force is applied. (a) and (b) show the initial straight beam (gray), the deformed shape under moment (target shape, blue) and when the moment is replaced by a force (red), as well as the optimized undeformed (light green) and deformed shapes (dark green).

\subsubsection{Beam with constant curvature}

Similar to the general setting in Sect. 5.1, we want to optimize an initially straight cantilever beam of length $L_{0}=1 \mathrm{~m}$ with a circular cross section of radius $r=0.025 \mathrm{~m}$, Young's modulus $E=10 \mathrm{MPa}$, and Poisson's ratio $\nu=0.5$. The beam is oriented along the $x$-direction and subject to an end-point moment $\hat{\mathbf{m}}(L)=(0,2,0)^{\top} \mathrm{Nm}$, which creates a deformed shape with constant (global) curvature $\kappa=0.652 \mathrm{~m}^{-1}$ around the $y$-axis.

This deformed configuration serves as target shape $\mathbf{r}_{t}$ for the optimization, for which we reduce the moment to 0 and instead apply an end-point force $\hat{\mathbf{n}}(L)=(0,-2,-3)^{\top} \mathrm{N}$. Using a parameterization of the centerline with $p_{0}=4, \ell_{0}=1, n_{0}=5, n_{u}=12$ and a discretization with $p=8, \ell=16, n=24$, we now optimize the initial centerline position such that the deformed shape with constant curvature is obtained.

The results of the optimization using the FA and IA gradient methods and SLSQP optimizer are shown in Fig. 7. In (a) and (b) the initial straight beam is shown in gray and the deformed target shape with constant curvature in blue. Replacing the moment with the force results in the deformed shape shown in red. After the optimization, which converged from an initial objective function value of $g=0.14$ to $g=2 \cdot 10^{-6}$ in only 16 iterations, see Fig. 7c, the optimized, now curved beam (light green) deforms into the desired shape (dark green, overlapping with blue).

With this example we could show that excellent convergence behavior can be achieved using our shape optimization approach for beams subject to large deformations and more complex loading scenarios, such as obtaining constant curvature when applying an end-point force.

\subsubsection{Post-buckling of curved beam}

Next, we introduce an example with even more complex large deformation behavior, i.e., softening of the force-displacement response and post-buckling. Such type of beam could, for instance, be part of a compliant mechanism or soft robotic structure, where a specific mechanical response behavior is required under loading.

The initial beam is parameterized using a B-Spline of degree 3 with 4 control points:

$$
\mathbf{r}_{0,1}=\left(\begin{array}{c}
10 \\
0 \\
0
\end{array}\right), \quad \mathbf{r}_{0,2}=\left(\begin{array}{c}
10 \\
10 \\
0
\end{array}\right), \quad \mathbf{r}_{0,3}=\left(\begin{array}{c}
10 \\
10 \\
-5
\end{array}\right), \quad \mathbf{r}_{0,4}=\left(\begin{array}{c}
0 \\
10 \\
-5
\end{array}\right)
$$




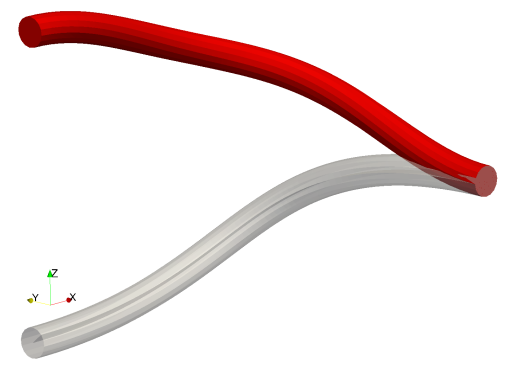

(a) initial

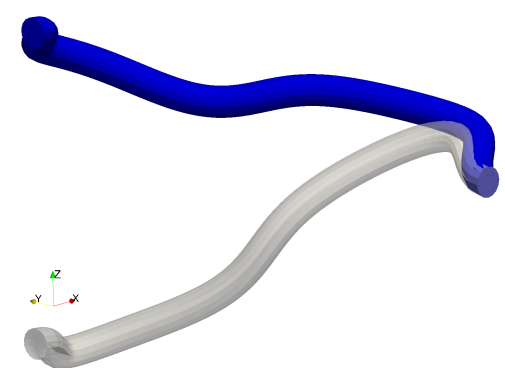

(b) SLSQP optimized

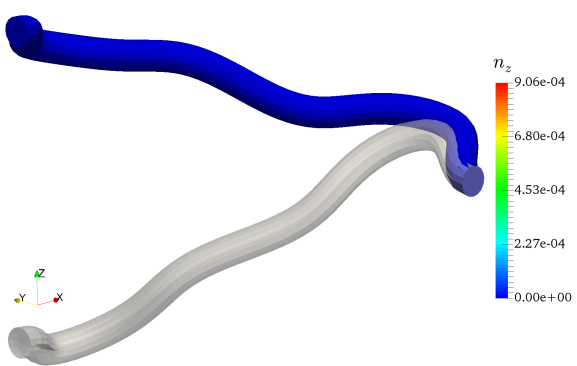

(c) MMA optimized

Figure 8: Displacement of initial and optimized 3D curved beams from their undeformed configurations (gray) to deformed, post-buckled shapes (colored by internal force $\mathbf{n}_{3}$ in $\mathrm{N}$ )

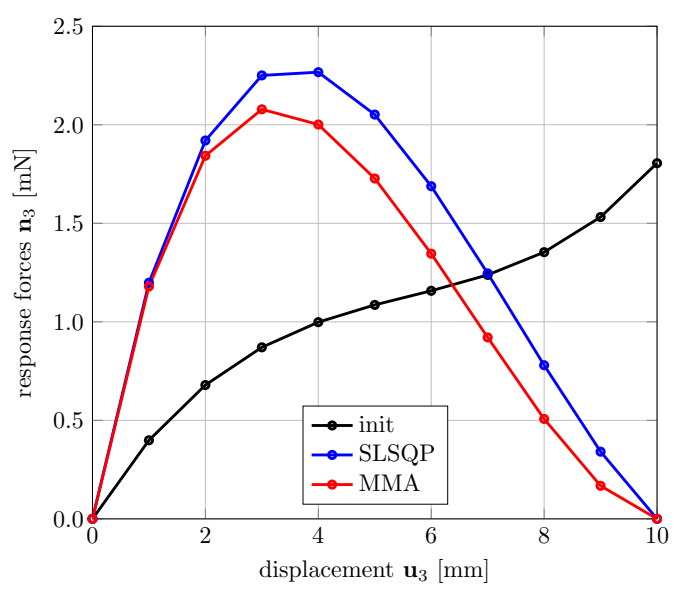

(a) force-displacement curves

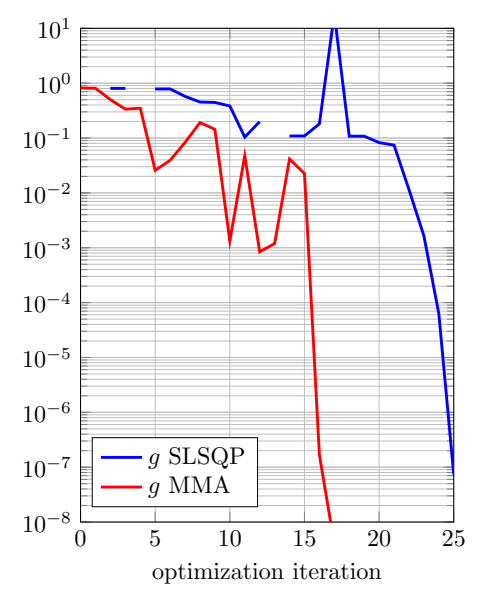

(b) convergence of objective function

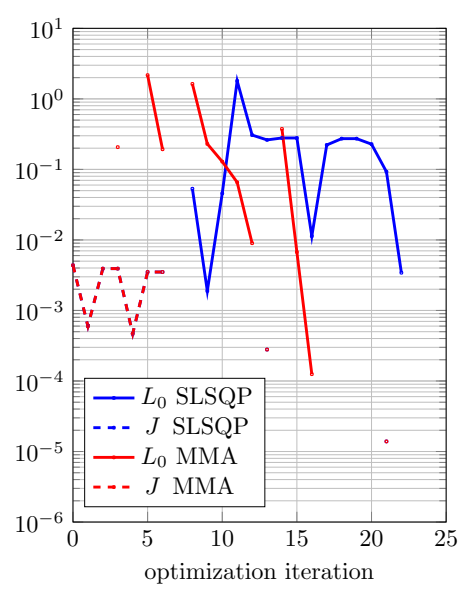

(c) convergence of constraints

Figure 9: Force-displacement curves of initial and optimized 3D curved beams in (a), as well as convergence of objective function and length and parameterization constraints in (b) and (c) for optimization using both SLSQP and MMA optimizers

and has a circular cross-section with radius $r=0.5 \mathrm{~mm}$, Young's modulus $E=1.0 \mathrm{MPa}$, and Poisson's ratio $\nu=0.45$. We assume that it represents a quarter of a symmetrical ring structure, where the end-points would be connected to the rest of the mechanism. Thus, both ends of the beam are clamped, but while displacements at $\xi=0$ are 0 , at $\xi=1$ a $z$-displacement of $10 \mathrm{~mm}$ is applied in a displacement-controlled simulation over 10 load steps with isogeometric discretization using $p=8, \ell=64$, see Fig. $8 \mathrm{a}$. Figure $9 \mathrm{a}$ shows the force-displacement response curves, summing the $z$-direction contributions of the internal forces from both end-points, indicating softening behavior.

Now, the objective is to minimize those response forces in $z$-direction, using the end-point force matching objective function type with $\mathbf{n}_{t}(0)=\mathbf{n}_{t}(L)=\mathbf{0}$ and weight $C_{n}=10^{6}$, see (44). Therefore, we refine the parameterization of the centerline to $p_{0}=4, \ell_{0}=8, n_{0}=12$. Since the beam is clamped at both ends, both the first and second control point at each end of the beam are excluded from the optimization and thus $n_{u}=3\left(n_{0}-4\right)=24$. Furthermore, to avoid mechanically infeasible centerline configurations, we introduce a speed of parameterization constraint for $J$, see Sect. 4.3.4, with $\varepsilon=0.2$ and factor $C_{J}=0.1$. Additionally, we also apply a maximum length constraint $L_{0} \leq 20 \mathrm{~mm}$, see Sect. 4.3.3, as the initial length is slightly smaller than $20 \mathrm{~mm}$. For both constraints, a tolerance of $\epsilon=0.001$ is used by the optimizers.

The results of the optimization using both SLSQP and MMA optimizers with the IA method are visualized in Fig. 8. Both, the optimized undeformed centerlines (gray) and the deformed beams (colored by internal force values $\mathbf{n}_{3}$ ) are shown for (b) the SLSQP and (c) the MMA optimizer. It can be seen 
that the two optimal shapes and their deformations are slightly different from each other. However, their force-displacement behavior is similar, including buckling with decreasing force magnitude for increasing deformation, see Fig. 9a. Most importantly, the force values $\mathbf{n}_{3}$ of both shapes at deformation $\mathbf{u}_{3}=10 \mathrm{~mm}$ are close to 0 . This is also indicated by the convergence plots of the objective function values and constraints in Fig. $9 \mathrm{~b}$ and Fig. $9 \mathrm{c}$, respectively, which show that $g$ is minimized from $g \approx 1$ to $g<10^{-7}$ within only 17 (MMA) and 25 (SLSQP) iterations.

Note that very high jumps and missing points in Fig. $9 \mathrm{~b}$ indicate un-converged attempts to solve $\overrightarrow{\mathbf{f}}(\overrightarrow{\mathbf{x}} ; \overrightarrow{\mathbf{u}})=$ $\mathbf{0}$, which means that the given beam configuration $\overrightarrow{\mathbf{u}}$ was not mechanically feasible. In those cases $g=\infty$ is returned to the optimizer and no gradient is computed. Missing points in Fig. 9c indicate that the constraint values were smaller than 0, i.e., the constraints were not violated. Furthermore, jumps to higher objective function values are related to "invalid" shapes that do not satisfy the constraints. Thus, the optimizers then typically move towards a less optimal, but more valid design in the next step.

Altogether, this numerical example shows the applicability of our shape optimization method for engineering applications of compliant beams subject to large deformations with highly nonlinear force-displacement behavior. Here, the optimized shapes exhibit buckling behavior, which could successfully reduce the applied force at the beam ends from $1.8 \mathrm{mN}$ to 0 .

\subsection{D auxetic metamaterial with prescribed Poisson's ratio}

Finally, we introduce an application of a 3D beam structure as a 3D truss lattice-like metamaterial. In recent years, metamaterials have received increasing attention from the applied mechanics community, especially for achieving soft, nonlinear, or functional behavior. Here, we want to optimize a 3D beam structure to engineer a metamaterial at the microstructural level with negative effective Poisson's ratios of $\nu_{12}=\nu_{13}=\hat{\nu}=-0.80$ in compression.

The design of our microstructure is inspired by the $2 \mathrm{D}$ auxetic metamaterials presented and optimized in [14. We model an eighth of a symmetric, cubic metamaterial unit-cell with a total of 48 rods, see Fig. 10a. with cross-section radius $r=0.5 \mathrm{~mm}$, Young's modulus $E=0.6 \mathrm{MPa}$, and Poisson's ratio $\nu=0.45$. The eighth has a width $W=10 \mathrm{~mm}$ and contains 6 rods, which are connected at the center of the cell and initially parameterized by quadratic B-Splines. The three control points of each rod are given by permutations of the following list of control points,

$$
\mathbf{r}_{0,1}=\left(\begin{array}{l}
0 \\
0 \\
0
\end{array}\right), \quad \mathbf{r}_{0,2}=\left(\begin{array}{c}
0 \\
-0.5 W+\Delta \\
0.5 W-\Delta
\end{array}\right), \quad \mathbf{r}_{0,3}=\left(\begin{array}{c}
-0.5 W \\
-0.5 W+\Delta \\
0.5 W-\Delta
\end{array}\right)
$$

where $\Delta<0.5 W$ is a parameter that controls the curvature of the initial design and here we use $\Delta=W / 4=$ $2.5 \mathrm{~mm}$

To determine the Poisson's ratios of the metamaterial, we perform a uniaxial compression simulation with an applied, macroscopic strain of $\varepsilon_{11}=-0.1$ in $x$-direction and assume that the unit-cell is subject to periodic boundary conditions. Thus, we apply prescribed $x$-displacements $\mathbf{u}_{1}= \pm \varepsilon_{11} W / 2$ to the ends of the two rods that are oriented in $x$-direction and anti-periodic boundary conditions to the other spatial directions of those ends and all other rod ends that lie on the boundary of the cell. Displacements at the interface of all 6 rods at the center of the cell are fixed (to restrict rigid body motions), but rotations are coupled through the interface conditions. The simulation is carried out incrementally over 8 load steps with a refined isogeometric discretization of $p=8, \ell=64$. The result is visualized in Fig. $10 \mathrm{~b}$ and can be used to calculate the effective Poisson's ratios:

$$
\nu_{12}=-\frac{\varepsilon_{22}}{\varepsilon_{11}}=-\frac{\mathbf{u}_{2}}{0.5 W \varepsilon_{11}}, \quad \nu_{13}=-\frac{\varepsilon_{33}}{\varepsilon_{11}}=-\frac{\mathbf{u}_{3}}{0.5 W \varepsilon_{11}} .
$$

Here, we find $\mathbf{u}_{2}=-0.1396 \mathrm{~mm}$ and $\mathbf{u}_{3}=-0.1513 \mathrm{~mm}$, which yields $\nu_{12}=-0.28$ and $\nu_{13}=-0.30$.

To optimize the metamaterial to yield the desired Poisson's ratio of $\hat{\nu}=-0.80$, we formulate an end-point matching-type objective function, where the target $y$ - and $z$-displacements should be $\mathbf{u}_{2}=\mathbf{u}_{3}=\hat{\nu} \varepsilon_{11} W / 2=$ $-0.4 \mathrm{~mm}$. For the shape optimization, we use a refined parameterization with $p_{0}=3, \ell_{0}=4, n_{0}=7$. 


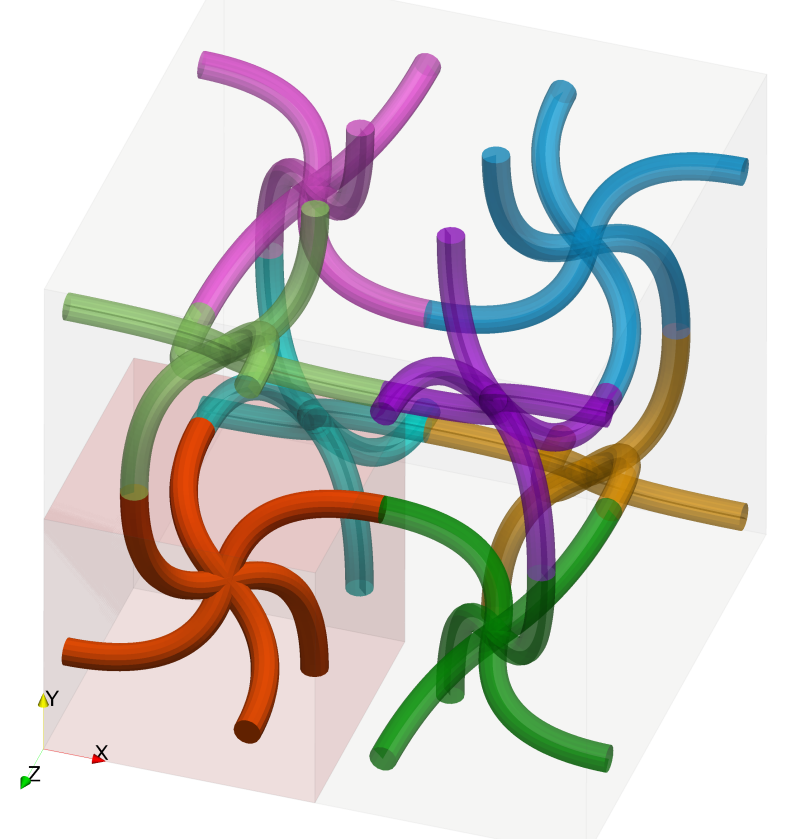

(a) metamaterial unit cell and simulated eighth (highlighted in red)
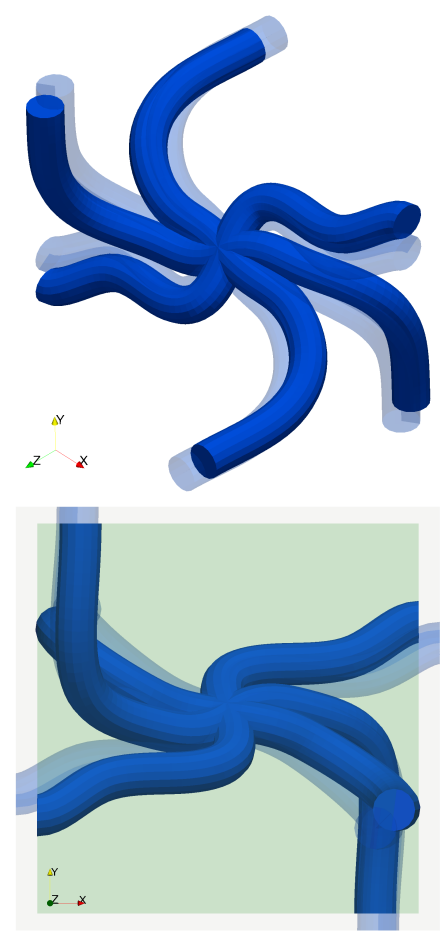

(c) optimized shape and deformation
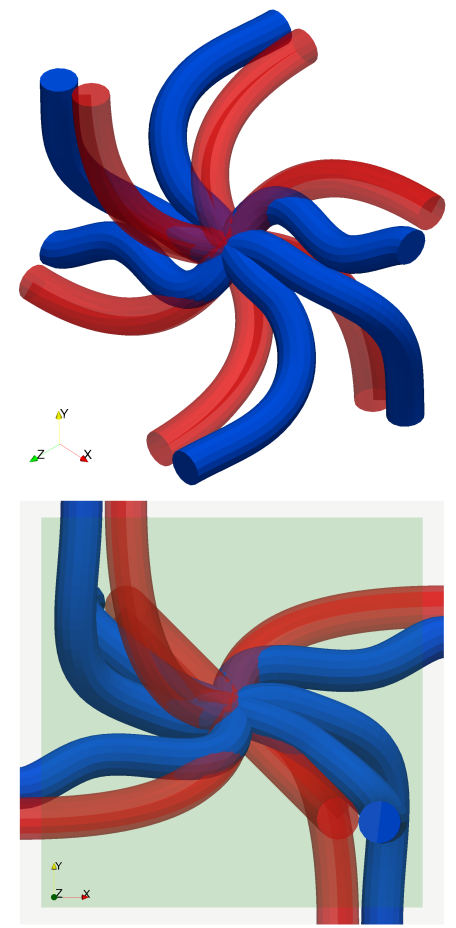

(d) initial vs. optimized shapes
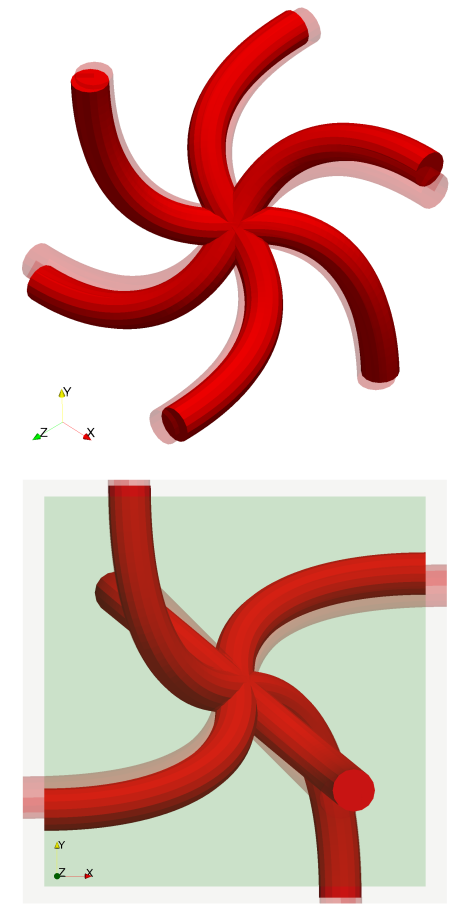

(b) initial shape and deformation
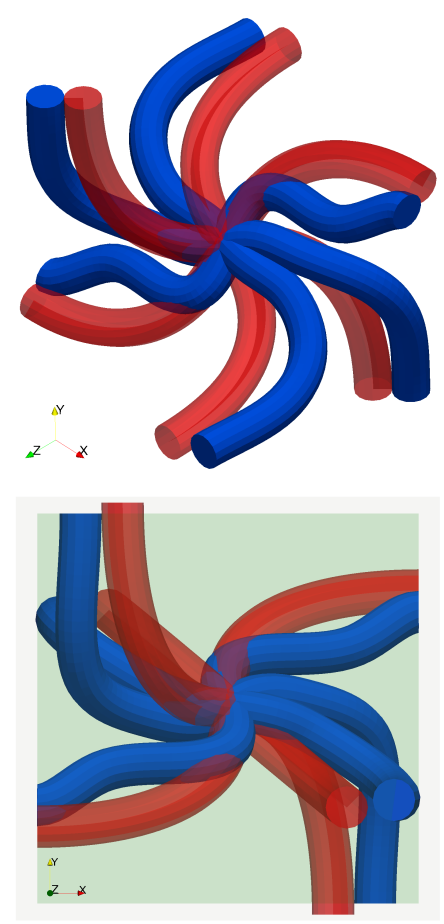

(e) initial vs. optimized deformation

Figure 10: Shape optimization of auxetic metamaterial with Poisson's ratio $\nu=-0.80$. In (b) and (c) the initial and optimized undeformed configurations are shown as transparent and the deformed configurations opaque. In (d) and (e) the optimized configurations with $\nu_{12}=\nu_{13}=-0.80$ (blue) are compared against the initial configurations with $\nu_{12}=-0.28, \nu_{13}=-0.30$ (transparent red). The gray boxes indicate the initial unit cell and the green boxes the deformed unit cell with $\nu=-0.80$ 


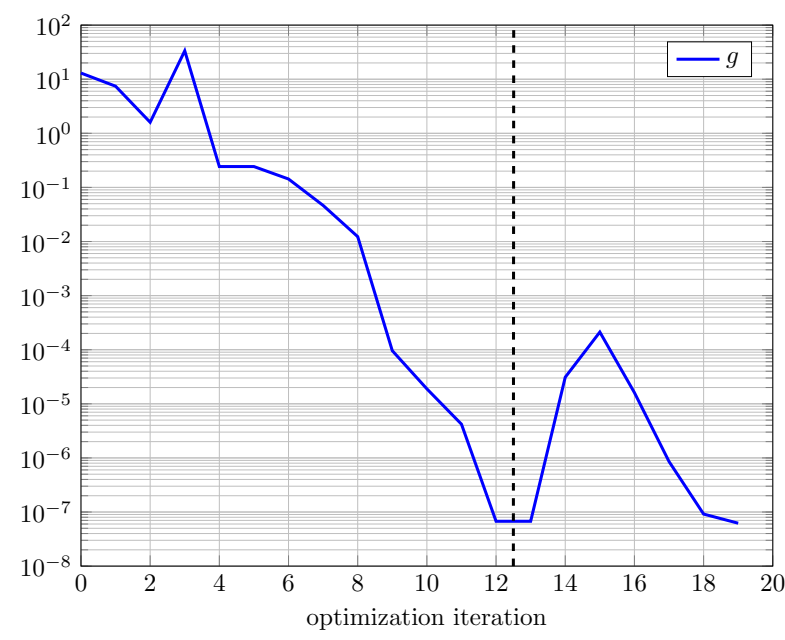

(a) convergence of objective function

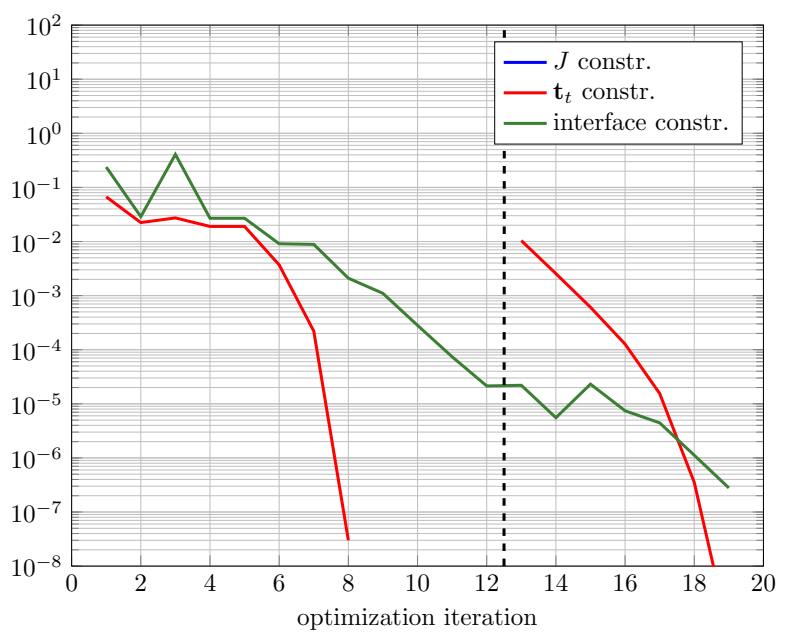

(b) convergence of constraints

Figure 11: Convergence of objective function and constraints for shape optimization of auxetic metamaterial with Poisson's ratio $\nu=-0.80$. After 12 iterations, the optimization is re-started with stricter enforcement of tangential and interface constraints

To maintain the size of the cell, the end points that interface with the boundary are only allowed to move within the boundary plane. Furthermore, we add a speed of parameterization constraint for $J$ with $\varepsilon=0.25$, interface constraints for the center interface as well as periodic boundary conditions with $\varepsilon=10^{\circ}$, and directional constraints at the boundary ends with $\varepsilon=0.001$ to ensure that tangents remain orthogonal to the boundary planes. The constraints have weights $C_{J}=C_{S}=C_{T}=0.1$ and the objective function $C_{r}=100$. In total, we have $n_{u}=102$ design variables and $n_{x}=2980$ discretization variables.

Now, the shape optimization problem is solved with the IA method and SLSQP optimizer. After only 12 iterations, the objective function value has dropped from $g=1.3$ to $g=6.7 \cdot 10^{-8}$ and the constraints are fulfilled up to the specified tolerance of $10^{-3}$, see Fig. 11. However, the tolerance of the interface constraints was specified quite generously with $\varepsilon=10^{\circ}$, so we restart the optimizer from the current optimum with a stricter tolerance of $\varepsilon=1^{\circ}$ and also decrease $C_{T}$ to 0.01 . After another 7 iterations, the optimization has converged with a minimal objective function value $g=6.2 \cdot 10^{-8}$. Now, we obtain $\mathbf{u}_{2}=-0.40 \mathrm{~mm}$ and $\mathbf{u}_{3}=-0.40 \mathrm{~mm}$, which yields the desired result $\nu_{12}=-0.80$ and $\nu_{13}=-0.80$. The optimal shape and deformation of the 3D auxetic metamaterial are visualized in Fig. 10c and compared against the initial shape in Fig. 10d and 10e. Overall, this shows a successful application of shape optimization of a nonlinear beam structure.

\section{Summary and conclusions}

We have introduced an isogeometric shape optimization method for nonlinear 3D beams. Beam centerlines are parameterized using spline curves and then an isogeometric collocation method is applied to discretize the geometrically exact 3D beam model. The benefits of IGA in terms of a unified description of geometry and discretization are exploited, as the control points of the centerline parameterization, which describe the shape of the beam, serve directly as design variables of the shape optimization approach. For solving the resulting nonlinear optimization problem using gradient-based optimizers and the adjoint method, we derive an incomplete/inconsistent sensitivity matrix that neglects the dependency of the initial cross-section orientations on the design variables, as well as a fully analytical sensitivity matrix that requires solution of another adjoint problem. We verify these approaches numerically against semi-analytical sensitivities and fully numerical gradient evaluation, and show that they can be successfully used to drive 
the gradient-based optimization. With our numerical applications we furthermore validate this unique 3D beam shape optimization method and show its versatility.

In the future, we would like to combine this shape optimization approach with design optimization of cross-section parameters, e.g., radius or Young's modulus, and ground structure topology optimization. This will allow to fully exploit the capabilities of advanced manufacturing technologies and support engineering design, e.g., of advanced metamaterials or compliant structures. However, adding more design freedom makes the resulting optimization problems more difficult to solve, since they are not convex anymore. This is, in general, already the case for the shape optimization problems studied here, and thus one needs to make sure the design parameterization is not too much refined, which may cause the optimizer to be trapped in local minima, or add suitable constraints. Thus, further work needs also to be spent on advancing optimization strategies for such complex, multi-disciplinary optimization problems.

\section{Acknowledgements}

The authors acknowledge support from the SUTD Digital Manufacturing and Design (DManD) Centre, supported by the Singapore National Research Foundation. Furthermore, we want to thank Dr. Narasimha Boddeti from SUTD for helpful discussions and the anonymous reviewers for their feedback and suggestions.

\section{Bibliography}

[1] N. Olhoff and J.E. Taylor. On structural optimization. Journal of Applied Mechanics, 50:1139, 1983.

[2] I. Gibson, D. Rosen, and B. Stucker. Additive Manufacturing Technologies: 3D Printing, Rapid Prototyping, and Direct Digital Manufacturing. Springer-Verlag New York, second edition, 2015.

[3] Q. Ge, C.K. Dunn, H.J. Qi, and M.L. Dunn. Active origami by 4D printing. Smart Materials and Structures, 23(9):094007, 2014.

[4] O. Weeger, Y.S.B. Kang, S.-K. Yeung, and M.L. Dunn. Optimal design and manufacture of active rod structures with spatially variable materials. 3D Printing and Additive Manufacturing, 3(4):204-215, 2016.

[5] Z. Ding, C. Yuan, X. Peng, T. Wang, H.J. Qi, and M.L. Dunn. Direct 4D printing via active composite materials. Science Advances, 3(4):e1602890, 2017.

[6] Z. Ding, O. Weeger, H.J. Qi, and M.L. Dunn. 4D rods: 3D structures via programmable 1D composite rods. Materials \& Design, 137:256-265, 2018.

[7] D. Rus and M.T. Tolley. Design, fabrication and control of soft robots. Nature, 521:467-475, 2015.

[8] N. Hu and R. Burgueõ. Buckling-induced smart applications: Recent advances and trends. Smart Materials and Structures, 24(6):063001, 2015.

[9] T.W. Murphey. Large strain composite materials in deployable space structures. In 17th International Conference on Composite Materials, Edinburgh, United Kingdom, 2009. The British Composites Society.

[10] A.D.B.L. Ferrera, P.R.O. Nóvoa, and A.T. Marques. Multifunctional material systems: A state-of-the-art review. Composite Structures, 151:3-35, 2016.

[11] N. Boddeti, Z. Ding, S. Kaijima, K. Maute, and M.L. Dunn. Simultaneous digital design and additive manufacture of structures and materials. Scientifc Reports, 8:15560, 2018.

[12] S. Babaee, J. Shim, J.C. Weaver, E.R. Chen, N. Patel, and K. Bertoldi. 3D soft metamaterials with negative Poisson's ratio. Advanced Materials, 25(36):5044-5049, 2013.

[13] X. Zheng, H. Lee, T.H. Weisgraber, M. Shusteff, J. DeOtte, E.B. Duoss, J.D. Kuntz, M.M. Biener, Q. Ge, J.A. Jackson, S.O. Kucheyev, N.X. Fang, and C.M. Spadaccini. Ultralight, ultrastiff mechanical metamaterials. Science, 344(6190):1373$1377,2014$.

[14] A. Clausen, F. Wang, J.S. Jensen, O. Sigmund, and J.A. Lewis. Topology optimized architectures with programmable poisson's ratio over large deformations. Advanced Materials, 27:5523-5527, 2015.

[15] B.G. Compton and J.A. Lewis. 3D-printing of lightweight cellular composites. Advanced Materials, 26(34):5930-5935, 2014.

[16] O. Weeger, N. Boddeti, S.-K. Yeung, S. Kaijima, and M.L. Dunn. Digital design and nonlinear simulation for additive manufacturing of soft lattice structures. Additive Manufacturing. under review.

[17] S.S. Antman. Nonlinear Problems of Elasticity, volume 107 of Applied Mathematical Sciences. Springer New York, 2005.

[18] S. Eugster. Geometric Continuum Mechanics and Induced Beam Theories, volume 75 of Lecture Notes in Applied and Computational Mechanics. Springer International Publishing, 2015.

[19] E. Reissner. On finite deformations of space-curved beams. Zeitschrift für angewandte Mathematik und Physik ZAMP, 32(6):734-744, 1981.

[20] J.C. Simo. A finite strain beam formulation. The three-dimensional dynamic problem. Part I. Computer Methods in Applied Mechanics and Engineering, 49(1):55-70, 1985.

[21] R.T. Haftka and R.V. Grandhi. Structural shape optimization-a survey. Computer Methods in Applied Mechanics and Engineering, 57(1):91-106, 1986. 
[22] Y. Ding. Shape optimization of structures: a literature survey. Computers E Structures, 24(6):985-1004, 1986.

[23] M.P. Saka and M. Ulker. Optimum design of geometrically nonlinear space trusses. Computers E Structures, 41(6):1387$1396,1991$.

[24] H. Madah and O. Amir. Truss optimization with buckling considerations using geometrically nonlinear beam modeling. Computers \& Structures, 192:233-247, 2017.

[25] N.V. Banichuk. Problems and Methods of Optimal Structural Design, volume 26 of Mathematical Concepts and Methods in Science and Engineering. Springer, 1983.

[26] A.P. Nagy, M.M. Abdalla, and Z. Gürdal. Isogeometric sizing and shape optimisation of beam structures. Computer Methods in Applied Mechanics and Engineering, 199(17-20):1216-1230, 2010.

[27] G. Radaelli and J.L. Herder. Isogeometric shape optimization for compliant mechanisms with prescribed load paths. In ASME International Design Engineering Technical Conferences and Computers and Information in Engineering Conference, volume 5A: 38th Mechanisms and Robotics Conference, 2014.

[28] N. Olhoff and R.H. Plaut. Bimodal optimization of vibrating shallow arches. International Journal of Solids and Structures, 19(6):553-570, 1983.

[29] A.P. Nagy, M.M. Abdalla, and Z. Gürdal. Isogeometric design of elastic arches for maximum fundamental frequency. Structural and Multidisciplinary Optimization, 43:135-149, 2011.

[30] T.J.R. Hughes, J.A. Cottrell, and Y. Bazilevs. Isogeometric analysis: CAD, finite elements, NURBS, exact geometry and mesh refinement. Computer Methods in Applied Mechanics and Engineering, 194(39-41):4135-4195, 2005.

[31] L.A. Piegl and W. Tiller. The NURBS Book. Monographs in Visual Communication. Springer, 1997.

[32] F. Auricchio, L. Beirão da Veiga, T.J.R. Hughes, A. Reali, and G. Sangalli. Isogeometric collocation methods. Mathematical Models and Methods in Applied Sciences, 20(11):2075-2107, 2010.

[33] A. Reali and T.J.R. Hughes. An introduction to isogeometric collocation methods. In G. Beer and S. Bordas, editors, Isogeometric Methods for Numerical Simulation, volume 561 of CISM International Centre for Mechanical Sciences, pages 173-204. Springer, 2015.

[34] D. Schillinger, J.A. Evans, A. Reali, M.A. Scott, and T.J.R. Hughes. Isogeometric collocation: Cost comparison with Galerkin methods and extension to adaptive hierarchical NURBS discretizations. Computer Methods in Applied Mechanics and Engineering, 267:170-232, 2013.

[35] F. Auricchio, L. Beirão da Veiga, J. Kiendl, C. Lovadina, and A. Reali. Locking-free isogeometric collocation methods for spatial Timoshenko rods. Computer Methods in Applied Mechanics and Engineering, 263:113-126, 2013.

[36] J. Kiendl, F. Auricchio, T.J.R. Hughes, and A. Reali. Single-variable formulations and isogeometric discretizations for shear deformable beams. Computer Methods in Applied Mechanics and Engineering, 284:988-1004, 2015.

[37] F. Auricchio, L. Beirão da Veiga, J. Kiendl, C. Lovadina, and A. Reali. Isogeometric collocation mixed methods for rods. Discrete and Continuous Dynamical Systems - Series S, 9(1):33-42, 2016.

[38] O. Weeger, S.-K. Yeung, and M.L. Dunn. Isogeometric collocation methods for Cosserat rods and rod structures. Computer Methods in Applied Mechanics and Engineering, 316:100-122, 2017.

[39] J. Kiendl, F. Auricchio, L. Beirão da Veiga, C. Lovadina, and A. Reali. Isogeometric collocation methods for the ReissnerMindlin plate problem. Computer Methods in Applied Mechanics and Engineering, 284:489-507, 2015.

[40] J. Kiendl, E. Marino, and L. De Lorenzis. Isogeometric collocation for the Reissner-Mindlin shell problem. Computer Methods in Applied Mechanics and Engineering, 325:645-665, 2017.

[41] F. Maurin, F. Greco, L. Coox, D. Vandepitte, and W. Desmet. Isogeometric collocation for Kirchhoff-Love plates and shells. Computer Methods in Applied Mechanics and Engineering, 329:396-420, 2018.

[42] F. Auricchio, L. Beirão da Veiga, T.J.R. Hughes, A. Reali, and G. Sangalli. Isogeometric collocation for elastostatics and explicit dynamics. Computer Methods in Applied Mechanics and Engineering, 249-252:2-14, 2012. Higher Order Finite Element and Isogeometric Methods.

[43] R. Kruse, N. Nguyen-Thanh, L. De Lorenzis, and T.J.R. Hughes. Isogeometric collocation for large deformation elasticity and frictional contact problems. Computer Methods in Applied Mechanics and Engineering, 296:73-112, 2015.

[44] M.-C. Hsu, C. Wang, A.J. Herrema, D. Schillinger, A. Ghoshal, and Y. Bazilevs. An interactive geometry modeling and parametric design platform for isogeometric analysis. Computers \& Mathematics with Applications, 70(7):1481-1500, 2015.

[45] J. Benzaken, A.J. Herrema, M.-C. Hsu, and J.A. Evans. A rapid and efficient isogeometric design space exploration framework with application to structural mechanics. Computer Methods in Applied Mechanics and Engineering, 316:1215$1256,2016$.

[46] A.J. Herrema, N.M. Wiese, C.N. Darling, B. Ganapathysubramanian, A. Krishnamurthy, and M.-C. Hsu. A framework for parametric design optimization using isogeometric analysis. Computer Methods in Applied Mechanics and Engineering, 316:944-965, 2017.

[47] V. Braibant and C. Fleury. Shape optimal design using B-splines. Computer Methods in Applied Mechanics and Engineering, 44(3):247-267, 1984.

[48] N. Olhoff, M.P. Bendsøe, and J. Rasmussen. On CAD-integrated structural topology and design optimization. Computer Methods in Applied Mechanics and Engineering, 89(1-3):259-279, 1991.

[49] U. Schramm and W.D. Pilkey. The coupling of geometric descriptions and finite elements using NURBs - A study in shape optimization. Finite Elements in Analysis and Design, 15:11-34, 1993.

[50] U. Schramm, W. Pilkey, R.I. DeVries, and M.P. Zebrowski. Shape design for thin-walled beam cross-sections using rational B-splines. AIAA Journal, 33(11):2205-2211, 1995.

[51] W.A. Wall, M.A. Frenzel, and C. Cyron. Isogeometric structural shape optimization. Computer Methods in Applied Mechanics and Engineering, 197(33-40):2976-2988, 2008. 
[52] B. Hassani, S.M. Tavakkoli, and N.Z. Moghadam. Application of isogeometric analysis in structural shape optimization. Scientia Iranica, 18(4):846-852, 2011.

[53] S. Cho and S.-H. Ha. Isogeometric shape design optimization: exact geometry and enhanced sensitivity. Structural and Multidisciplinary Optimization, 38, 2009.

[54] X. Qian. Full analytical sensitivities in NURBS based isogeometric shape optimization. Computer Methods in Applied Mechanics and Engineering, 199(29-32):2059-2071, 2010.

[55] J. Kiendl, R. Schmidt, R. Wüchner, and K.-U. Bletzinger. Isogeometric shape optimization of shells using semi-analytical sensitivity analysis and sensitivity weighting. Computer Methods in Applied Mechanics and Engineering, 274:148-167, 2014.

[56] D. Fußeder, B. Simeon, and A.-V. Vuong. Fundamental aspects of shape optimization in the context of isogeometric analysis. Computer Methods in Applied Mechanics and Engineering, 286, 2015.

[57] S.F. Hosseini, B. Moetakef-Imani, S. Hadidi-Moud, and B. Hassani. Pre-bent shape design of full free-form curved beams using isogeometric method and semi-analytical sensitivity analysis. Structural and Multidisciplinary Optimization, 2018.

[58] H. Lian, P. Kerfriden, and S.P.A. Bordas. Shape optimization directly from CAD: An isogeometric boundary element approach using T-splines. Computer Methods in Applied Mechanics and Engineering, 317:1-41, 2017.

[59] C. Wang, S. Xia, X. Wang, and X. Qian. Isogeometric shape optimization on triangulations. Computer Methods in Applied Mechanics and Engineering, 331:585-622, 2018.

[60] K. Bandara and F. Cirak. Isogeometric shape optimisation of shell structures using multiresolution subdivision surfaces. Computer-Aided Design, 95:62-71, 2018.

[61] Z.-P. Wang, L.H. Poh, J. Dirrenberger, Y. Zhu, and S. Forest. Isogeometric shape optimization of smoothed petal auxetic structures via computational periodic homogenization. Computer Methods in Applied Mechanics and Engineering, 323:250-271, 2017.

[62] A.P. Nagy, S.T. IJsselmuiden, and M.M. Abdalla. Isogeometric design of anisotropic shells: Optimal form and material distribution. Computer Methods in Applied Mechanics and Engineering, 264:145-162, 2013.

[63] C. Anitescu, Y. Jia, Y.J. Zhang, and T. Rabczuk. An isogeometric collocation method using superconvergent points. Computer Methods in Applied Mechanics and Engineering, 284:1073-1097, 2015.

[64] H. Gomez and L. De Lorenzis. The variational collocation method. Computer Methods in Applied Mechanics and Engineering, 309:152-181, 2016.

[65] B. Jüttler, U. Langer, A. Mantzaflaris, S.E. Moore, and W. Zulehner. Geometry + Simulation Modules: Implementing Isogeometric Analysis. PAMM, 14(1):961-962, 2014.

[66] R.T. Haftka and Z. Gürdal. Elements of Structural Optimization, volume 11 of Solid Mechanics and Its Applications. Springer Netherlands, third edition, 1992.

[67] R. Fletcher. Practical Methods of Optimization. John Wiley \& Sons, second edition, 1987.

[68] R. Siegbert, J. Kitschke, H. Djelassi, M. Behr, and S. Elgeti. Comparing optimization algorithms for shape optimization of extrusion dies. Proceedings in Applied Mathematics and Mechanics, 14, 2014.

[69] S.G. Johnson. The NLopt nonlinear-optimization package, https://nlopt.readthedocs.io/,

[70] J.A. Cottrell, A. Reali, Y. Bazilevs, and T.J.R. Hughes. Isogeometric analysis of structural vibrations. Computer Methods in Applied Mechanics and Engineering, 195(41-43):5257-5296, 2006. 